\title{
Positivity-preserving cell-centered Lagrangian schemes for multi-material compressible flows: From first-order to high-orders. Part II: The two-dimensional case
}

\author{
François Vilar ${ }^{\mathrm{a}, *}$, Chi-Wang Shu ${ }^{\mathrm{a}}$, Pierre-Henri Maire ${ }^{\mathrm{b}}$ \\ ${ }^{a}$ Division of Applied Mathematics, Brown University, Providence, RI 02912, USA \\ ${ }^{b}$ CEA/CESTA, 15 Avenue des Sablières CS 600133116 Le Barp cedex France
}

\begin{abstract}
This paper is the second part of a series of two. It follows [44], in which the positivity-preservation property of methods solving one-dimensional Lagrangian gas dynamics equations, from first-order to high-orders of accuracy, was addressed. This article aims at extending this analysis to the two-dimensional case. This study is performed on a general first-order cell-centered finite volume formulation based on polygonal meshes defined either by straight line edges, conical edges, or any high-order curvilinear edges. Such formulation covers the numerical methods introduced in $[6,32,5,41,43]$. This positivity study is then extended to high-orders of accuracy. Through this new procedure, scheme robustness is highly improved and hence new problems can be tackled. Numerical results are provided to demonstrate the effectiveness of these methods. It important to point out that even if this paper is concerned with purely Lagrangian schemes, the theory developed is of fundamental importance for any methods relying on a purely Lagrangian step, as ALE methods or non-direct Euler schemes.

Keywords: positivity-preserving high-order methods, cell-centered Lagrangian schemes, updated and total Lagrangian formulations, Godunov-type method, unstructured moving grid, multi-material compressible flows, equations of state, Riemann solver
\end{abstract}

\footnotetext{
* Corresponding author

Email addresses: francois_vilar@brown.edu (François Vilar), shu@dam.brown.edu (Chi-Wang Shu), maire@celia.u-bordeaux1.fr (Pierre-Henri Maire)
} 


\section{Introduction}

This paper is the second part of a series of two, which investigates the two-dimensional situation. The one-dimensional case has been addressed in the first paper, [44]. Here, we aim at demonstrating the positivity-preservation property of methods solving two-dimensional Lagrangian gas dynamics equations, from first-order to high-orders of accuracy, under suitable constraints.

When we mention Lagrangian gas dynamics, we refer to the kinematic description which consider a time dependent reference frame that follows the fluid motion, $[19,18]$. Such formalism is particularly well adapted to describe the time evolution of fluid flows contained in regions undergoing large shape changes, or the simulation of multi-material compressible flows. In the Lagrangian description, the gas dynamics system may be derived in two different but consistent formulations, namely the updated Lagrangian formulation based on the moving configuration, and the total Lagrangian formulation based on the fixed initial configuration. In this latter approach, the physical conservation laws are written employing the Lagrangian coordinates which refer to the initial configuration of the fluid flow. We refer to [19, 39, 43] for further details on this particular description. In contrast to the total Lagrangian formulation, the updated Lagrangian formulation is a moving domain method, in which the gas dynamics equations are written employing the moving Eulerian coordinates. They refer to the current configuration of the fluid flow. And to solve updated Lagrangian gas dynamics equations, two approaches are mainly employed, namely the cell-centered and staggered approaches. Let us however mention that besides these two frameworks, a third one, referred to as point-centered hydrodynamic, has recently grows quickly in popularity these past years. For a precise description of these approaches, along with a relatively complete overview of Lagrangian schemes current status, we refer to the introduction of our paper concerned with the 1D, [44], and the references within.

The issue of robustness is fundamental for numerical schemes. Considering gas dynamics for instance, numerical approximations may generate negative density or pressure, which may lead to nonlinear instability or crash of the code. This phenomenon is even more critical using a Lagrangian formalism, the grid moving and being deformed during the calculation. Furthermore, most of the problems studied in this framework contain very intense shock waves. And contrary to the Eulerian case where non-admissible states may appear in low density regions, in the Lagrangian frame it is in regions of high compression that the scheme may fail and produce negative specific volume or internal energy. These phenomena are the consequence of the lack of a particular property, often referred to as positively conservation or positivity preservation. This issue of positivity is generally addressed for the Eulerian case, see for instance [15, 37, 38, 3, 4], but very few papers exist on this topic for the Lagrangian formulation. Let us yet mention the works presented in $[35,17,9]$.

In this paper, the numerical schemes presented rely on a solver widely used in the Lagrangian community and generally referred to as the two-states solver, see $[13,30]$. This solver proved in our 1D paper, [44], to be positivity preserving under particular definition of the wavespeeds, or by means of an additional time step constraint. We thus extend in this article the positivity analysis to the two-dimensional frame. Then the question on how to extend this positivity property to higher-order accuracy is addressed. In the Eulerian framework, Zhang and Shu developed recently in a series of papers, [45, 46, 47, 48], a general technique to extend the positivity-preserving property to high-

order schemes based on finite-volume-like discretizations. This is the technique used in this paper to assess the positivity of the high-order Lagrangian schemes presented here. 
To this end, the remainder of this paper is organized as follows: In Section 2, we briefly recall the different forms of Lagrangian gas dynamics system of equations. Then, in Section 3, the different equations of state (EOS) employed to thermodynamically close these systems are presented. In Sections 4 and 5, we present a general first-order finite volume formulation that will prove to fit a wide range of existing cell-centered Lagrangian schemes. The scheme positivity-preserving property is addressed in Section 6. The spatial high-order extension of the introduced Lagrangian scheme and its related positivity-preserving analysis are respectively performed in Sections 7 and 8, while the particular limitation permitting the preservation of such property from the first-order to highorders is developed in Section 9. We end the theoretical analysis by presenting stability properties deriving from the positivity of the numerical solution, Section 10, and by a discussion on high-order time discretization, Section 11. Finally, numerical results, provided in Section 12, demonstrate the effectiveness of these methods.

Let us emphasize once more that this paper follows the one concerned with the one-dimensional case, [44]. Most of the ingredients and techniques used in the multi-dimensional case being based on those developed in the 1D case, we warmly recommend to first read [44] for sake of clarity.

\section{Governing equations}

We will not give in detail how to derive, from the Euler equations, the different Lagrangian gas dynamics systems. For a complete review of such process, the interested reader may refer to $[19,13,30,43]$. We will just recall in the remainder the gas dynamics system in both updated and total Lagrangian formulations. On a frame moving through the fluid flow, the two-dimensional gas dynamics governing equations write in an updated Lagrangian formulation as

$$
\rho \frac{\mathrm{d} \mathrm{U}}{\mathrm{d} t}+\nabla_{x} \cdot \mathrm{F}(\mathrm{U})=0
$$

where $\mathrm{U}=(\tau, \boldsymbol{u}, e)^{\mathrm{t}}$ is the vector of the mass conserved variables and $\mathrm{F}(\mathrm{U})=(-\boldsymbol{u}, \mathbb{1}(1) p, \mathbb{1}(2) p, p \boldsymbol{u})^{\mathrm{t}}$ the flux, with $\mathbb{1}(i)=\left(\delta_{i 1}, \delta_{i 2}\right)^{\mathrm{t}}$. Here, $\tau=\frac{1}{\rho}$ stands for the specific volume, while $\boldsymbol{u}, p$ and $e$ refer to as the velocity, pressure and specific total energy of the fluid under consideration. In (1), $\frac{\mathrm{d}}{\mathrm{d} t}$ is nothing but the material derivative, which corresponds to the temporal derivative along the trajectories, and is defined through

$$
\frac{\mathrm{d}}{\mathrm{d} t} f(\boldsymbol{x}, t)=\frac{\partial}{\partial t} f(\boldsymbol{x}, t)+\boldsymbol{u} \cdot \nabla_{x} f(\boldsymbol{x}, t),
$$

where $f$ is a fluid variable with sufficient smoothness. Definition (2) holds provided that the referential moves with the fluid velocity. This statement is emphasized in the trajectory equation, $\frac{\partial}{\partial t} \boldsymbol{x}(\boldsymbol{X}, t)=\boldsymbol{u}(\boldsymbol{x}(\boldsymbol{X}, t), t)$, where $\boldsymbol{x}(\boldsymbol{X}, t)$ is a fluid particle initially located at $\boldsymbol{X}$. Integrating equation (1) on a control volume $\omega$, and by means of mass conservation and the Reynolds transport formula $[19,30,43]$, one can easily get this system into its conservative integral form as

$$
\frac{\partial}{\partial t} \int_{\omega} \rho \mathrm{U} \mathrm{d} v+\int_{\partial \omega} \mathrm{F}(\mathrm{U}) \cdot \boldsymbol{n} \mathrm{d} s=0
$$

where $\boldsymbol{n}$ represents the unit outward normal of $\omega$ boundary. Now, the counterpart of system (1) on the initial referential, namely the system of gas dynamics equations in a total Lagrangian framework, reads as follows

$$
\rho^{0} \frac{\partial \mathrm{U}}{\partial t}+\nabla_{X} \cdot\left(|\mathrm{J}| \mathrm{J}^{-1} \mathrm{~F}(\mathrm{U})\right)=0
$$


where $\rho^{0}$ is the fluid initial density. In (4), J $=\nabla_{X} \boldsymbol{x}$ reads as the Jacobian of the fluid flow, also referred to as the deformation gradient tensor, and $|\mathrm{J}|$ is nothing but the determinant of $\mathrm{J}$. We assume $|\mathrm{J}|>0$ for the flow map to be invertible. Similarly to (3), one can write system (4) in an integral conservative form as

$$
\frac{\partial}{\partial t} \int_{\Omega} \rho^{0} \mathrm{U} \mathrm{d} V+\int_{\partial \Omega} \mathrm{F}(\mathrm{U}) \cdot|\mathrm{J}| \mathrm{J}^{-t} \boldsymbol{N} \mathrm{d} S=0
$$

where $\boldsymbol{N}$ represents the unit outward normal of the boundary of $\Omega$. Obviously, if $\omega$ is the image through the fluid flow of the initial volume $\Omega$, then $\boldsymbol{n}$ will be nothing but the image in the actual configuration of the initial normal $\boldsymbol{N}$. In this case, they are related to each other by means of the Nanson formula

$$
|\mathrm{J}| \mathrm{J}^{-t} \boldsymbol{N} \mathrm{d} S \equiv \mathrm{J}^{\star} \boldsymbol{N} \mathrm{d} S=\boldsymbol{n} \mathrm{d} s
$$

where $\mathrm{J}^{\star}$ reads as the cofactor matrix of tensor $\mathrm{J}$. By the use of Nanson formula (6) along with the mass conservation $\rho|\mathrm{J}|=\rho^{0}$, it is then obvious to see the perfect equivalence between the updated and total Lagrangian formulations, systems (3) and (5).

The thermodynamic closure of these systems is given by expressing the pressure $p$ and the temperature $T$ in terms of the density $\rho$, the specific entropy $S$, and the internal energy $\varepsilon=e-\frac{1}{2} \boldsymbol{u}^{2}$ through the equation of state

$$
p=\left.\rho^{2} \frac{\partial \varepsilon}{\partial \rho}\right|_{S} \quad \text { and } \quad T=\left.\frac{\partial \varepsilon}{\partial S}\right|_{\rho}
$$

This constitutive equation is consistent with the fundamental Gibbs relation

$$
T \mathrm{~d} S=\mathrm{d} \varepsilon+p \mathrm{~d} \tau .
$$

We also assume that the specific entropy is a concave function with respect to the specific volume $\tau$ and the internal energy $\varepsilon$. We note that the equation of state can also be written under the socalled incomplete form $p=p(\rho, \varepsilon)$. Finally, the thermodynamic sound speed is defined as $a^{2}=\left.\frac{\partial p}{\partial \rho}\right|_{S}$. Obviously, $a^{2}$ has to remain positive at all time. A numerical scheme not ensuring this property may lead to crash of the code. This remark will be one of the guiding principles of this paper, for the different equations of state studied.

\section{Equations of state}

In this paper, we consider several widely assessed multi-material problems with general equations of state. In practice, we make use of four different EOS, from the simple ideal gas one, to the more complex Mie-Grüneisen EOS for solids. These EOS are the ones used in the one-dimensional case. Thus, for further details, we refer to the appendix section of [44].

Gamma gas law. For perfect gas, we define the thermodynamic pressure as

$$
p=\rho(\gamma-1) \varepsilon
$$

where $\gamma>1$ is the polytropic index of the gas. 
Stiffened gas EOS. This equation of state, generally used for water under very high pressures, is more generic than the ideal one. Here, the pressure reads

$$
p=\rho(\gamma-1) \varepsilon-\gamma p_{s},
$$

where $p_{s}$ is a positive constant representing the molecular attraction between water molecules.

Jones-Wilkins-Lee (JWL) EOS. This equation of state is used to describe detonation-product gas in explosions. Here, the pressure reads

$$
p=\rho(\gamma-1) \varepsilon+f_{j}(\rho),
$$

where the definition of the positive function $f_{j}(\rho)$ can be found in [44].

Mie-Grüneisen EOS. Even if this paper is concerned with solving gas dynamics problems, one can decide to plug equations of state generally used in solid mechanics into the studied system. Here, we make use of the Mie-Grüneisen equation of state for shock-compressed solids. In this case, the pressure reads

$$
p=\rho_{0} \Gamma_{0} \varepsilon+\rho_{0} a_{0}^{2} f_{m}(\eta)
$$

where $\eta=\frac{\rho}{\rho_{0}}, \rho_{0}$ being the density of the unstressed material. The physical meaning of the constants involved here, as well as the definition of $f_{m}(\eta)$, can be found in [44].

These different definitions of pressure yield different domains of validity. Indeed, physically the fluid flow has the positivity property for some variables such as density, internal energy, or the quantity inside the square root to define the sound speed. Let us note that it is not always the case of the pressure, which can yield negative values for the stiffened gas EOS for instance. In the one-dimensional part of this work, [44], we have shown that a sufficient condition for the solution to be physical writes as follows

$$
\left.(\rho, \widehat{\varepsilon}) \in] \rho_{\min }, \rho_{\max }[\times] \varepsilon_{\min },+\infty\right]
$$

where $\widehat{\varepsilon}=\varepsilon-p_{s} \tau$ in the stiffened EOS case and $\widehat{\varepsilon}=\varepsilon$ otherwise, and $\rho_{\min }, \rho_{\max }$ and $\varepsilon_{\min }$ are positive constants depending on the EOS. Furthermore, if the solution lies in this validity domain then one can know for sure that $a^{2}>0$. That being said, we now aim at ensuring that the numerical

schemes under consideration produce solutions lying in this validity domain. But before exploring the case of a generic order of accuracy in space, one needs to ensure that the first-order scheme preserves the desired property. The first-order scheme will provide the healthy base on which one can build an high-order approximation.

\section{First-order scheme}

Using the same procedure as in the one-dimensional case, let $\Omega$ be the domain filled by the fluid in its initial configuration. Its image through the flow map is the considered domain $\omega$ at time $t$. These domains are partitioned into non-overlapping cells, respectively $\Omega_{c}$ and $\omega_{c}$, where $\omega_{c}$ is the image of cell $\Omega_{c}$ through the fluid flow. Then, integrating on $\omega_{c}$, respectively $\Omega_{c}$, the Lagrangian 
gas dynamics systems presented and applying a standard forward Euler scheme as time integrator, one gets the following first-order scheme

$$
\mathrm{U}_{c}^{n+1}=\mathrm{U}_{c}^{n}-\frac{\Delta t^{n}}{m_{c}} \int_{\partial \omega_{c}} \overline{\mathrm{F}} \cdot \boldsymbol{n} \mathrm{d} s,
$$

where $\mathrm{U}_{c}^{n}$ reads as the mass averaged value of the solution vector $\mathrm{U}$ as

$$
\mathrm{U}_{c}^{n}=\frac{1}{m_{c}} \int_{\Omega_{c}} \rho^{0} \mathrm{U} \mathrm{d} V=\frac{1}{m_{c}} \int_{\omega_{c}} \rho \mathrm{Ud} v
$$

with $m_{c}$ the constant mass of cell $\omega_{c}$. In equation $(12), \overline{\mathrm{F}}=(-\overline{\boldsymbol{u}}, \mathbb{1}(1) \bar{p}, \mathbb{1}(2) \bar{p}, \bar{p} \overline{\boldsymbol{u}})^{\mathrm{t}}$ reads for the numerical flux. Scheme (12) is said to be compatible, which means that both discretizations on the updated Lagrangian frame (1) and the total Lagrangian frame (4) are perfectly equivalent, under the assumptions that the Nanson formula is true at the discrete level, as well as the Piola compatibility condition ensured, see [43] for more details.

A wide number of cell-centered numerical schemes take their starting point from equation (12). The differences will then arise from the definition of the numerical flux $\overline{\mathrm{F}}$ and the treatment of the boundary integral $\int_{\partial \omega_{c}}$. Consequently, to avoid the positivity-preserving study to be too scheme specific, we present and make use here of a general first-order finite volume formulation that will prove to fit different existing cell-centered Lagrangian schemes, such as those presented in $[6,32,5$, 41, 43]. The general scheme relies on general polygonal cells defined either by straight line edges, Figure 1(a), conical edges, Figure 1(b), or any high-order curvilinear edges, Figure 1(c). By means

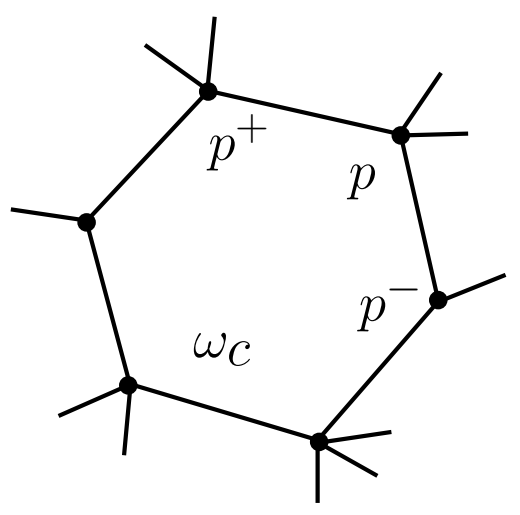

(a) Straight line edges.

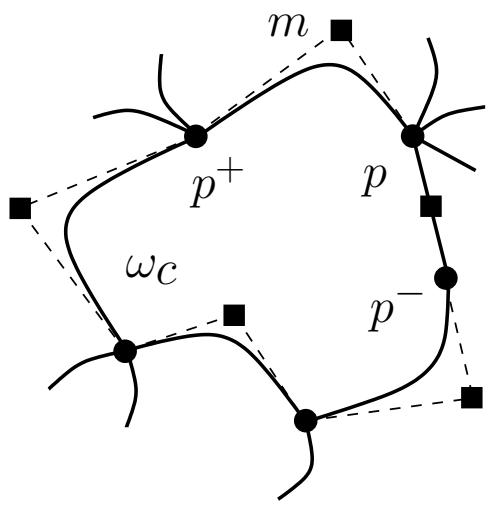

(b) Conical edges.

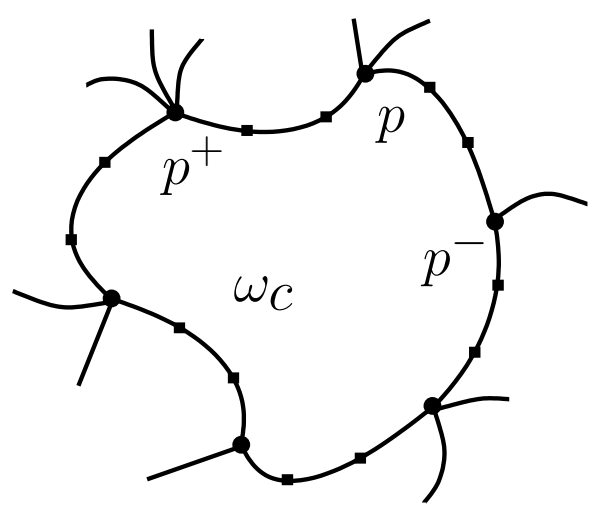

(c) Polynomial edges.

Figure 1: General polygonal cells.

of quadrature rules or some approximations, the cell boundary integral present in equation (12) can be expressed as a combination of control point contributions. Following this statement, the first-order scheme (12) can be expressed as follows

$$
\mathrm{U}_{c}^{n+1}=\mathrm{U}_{c}^{n}-\frac{\Delta t^{n}}{m_{c}} \sum_{q \in \mathcal{Q}_{c}} \overline{\mathrm{F}}_{q c} \cdot l_{q c} \boldsymbol{n}_{q c},
$$

where the $l_{q c} \boldsymbol{n}_{q c}$ correspond to some normals at time level $n$ to be defined. In (14), $\mathcal{Q}_{c}$ is the chosen control point set of cell $\omega_{c}$. This set has to contain the node set of the cell, i.e. $\mathcal{P}_{c} \subseteq \mathcal{Q}_{c}$. Indeed, 
the mesh has to be advected in some ways, and thus some velocity has to be allocated to all grid points. In equation (14), the control flux $\overline{\mathrm{F}}_{q c}=\left(-\overline{\boldsymbol{u}}_{q}, \mathbb{1}(1) \bar{p}_{q c}, \mathbb{1}(2) \bar{p}_{q c}, \bar{p}_{q c} \overline{\boldsymbol{u}}_{q}\right)^{\mathrm{t}}$ plays this role. Indeed, any grid control point $q$ will be advected through

$$
\boldsymbol{x}_{q}^{n+1}=\boldsymbol{x}_{q}^{n}+\Delta t^{n} \overline{\boldsymbol{u}}_{q} .
$$

Let us emphasize that $\overline{\mathrm{F}}_{q c}$, in addition to be local to the control point $q$, is local the cell $\omega_{c}$ under consideration. This is different from the classical finite volume framework where the numerical flux on the cell boundary enforce the scheme conservation, and consequently is continuous on the edge from one cell to another. The reason a discontinuous numerical flux is considered is, as indicated previously, the cornerstone of any Lagrangian scheme is to move the mesh. We would like to do so in a similar way as in the one-dimensional case, namely by means of Riemann solvers. However, even if for a control point on a face, Figure 2(a), the two neighboring cells allow us to use a 1D Riemann solver in the normal direction, this is no more the case for nodes, Figure 2(b).

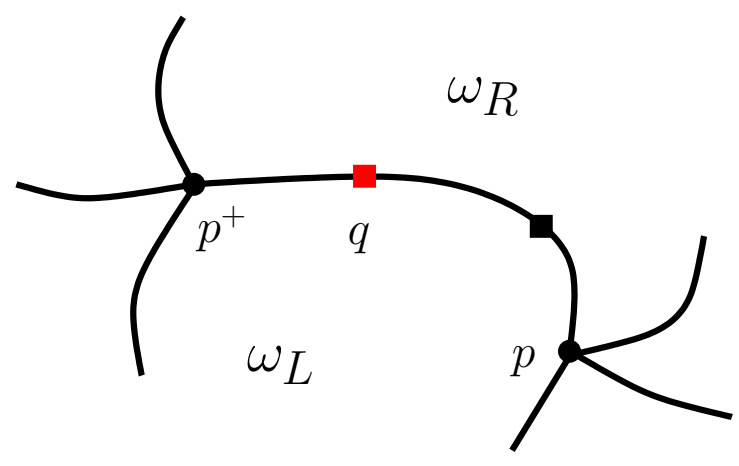

(a) Face point neighboring cells.

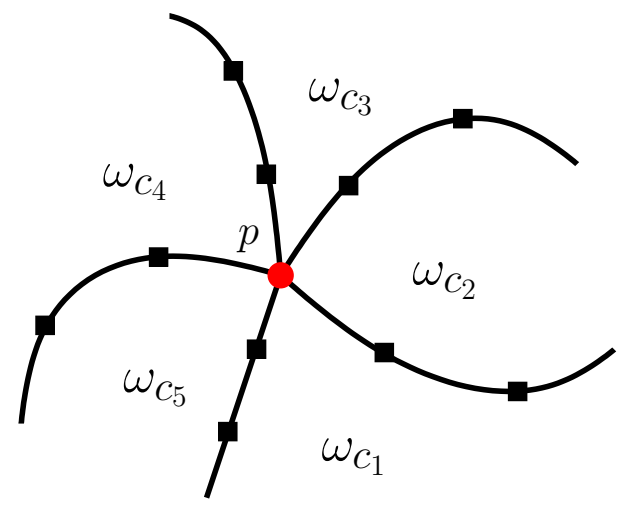

(b) Node neighboring cells.

Figure 2: Points neighboring cell sets.

In the $2 \mathrm{D}$ case, the numerical flux at node $p$ yields three unknowns, $\bar{p}_{p}$ the pressure and $\overline{\boldsymbol{u}}_{p}$ its velocity. However, applying any 1D Riemann solver on each edge surrounding $p$ will produce a too large number of equations. And employing a least square or any minimizing procedure, as it was done in [2], will leads to the loss of the one-dimensional scheme properties as the entropy production or positivity.

To overcome this difficulty, the idea introduced in the GLACE scheme, $[14,6]$, was to break the continuity of the numerical flux pressure $\bar{p}$. Indeed, the only strong continuity requirement lies on the velocity because we do not want the nodes to split. By means of this assumption, the control point pressure $\bar{p}_{q}$ becomes local to the cell $\omega_{c}$, namely $\bar{p}_{q c}$, and will be defined through the one-dimensional two-states solver, presented in our paper concerned with the 1D [44], as follows

$$
\bar{p}_{q c}=p_{c}^{n}-\widetilde{z}_{q c}\left(\overline{\boldsymbol{u}}_{q}-\boldsymbol{u}_{c}^{n}\right) \cdot \boldsymbol{n}_{q c},
$$

where $\widetilde{z}_{q c}>0$ is again a local approximation of the acoustic impedance. Different choices in the wave speeds $\widetilde{z}_{q c}$ lead to different schemes. The simplest one, and certainly the most widely used, is the acoustic approximation where the wave speeds are set to be the acoustic impedances, i.e. $\widetilde{z}_{q c} \equiv z_{c}^{n}=\rho_{c}^{n} a_{c}^{n}$. In this particular case, the 1D two-states solver is nothing but the Godunov 
acoustic solver.

Let us emphasize the pressure continuity relaxation obviously leads to the loss of the scheme conservation. Nonetheless, this property will be recovered by the construction of the scheme itself. For the sake of simplicity, we set ourselves in the simple case where no external contribution is applied on the domain boundary, i.e. $\int_{\partial \omega} \overline{\mathrm{F}} \cdot \boldsymbol{n} \mathrm{d} s=0$. In this particular case, the scheme conservation writes

$$
\sum_{c} m_{c} \mathrm{U}_{c}^{n+1}=\sum_{c} m_{c} \mathrm{U}_{c}^{n}
$$

And substituting scheme (14) in relation (17) leads to following condition on the numerical pressures

$$
\sum_{c} \sum_{q \in \mathcal{Q}_{c}} \bar{p}_{q c} l_{q c} \boldsymbol{n}_{q c}=\mathbf{0}
$$

In the end, satisfying such relation will allow us to define explicitly the velocity for grid control points. Finally, the differences in the first-order schemes presented for instance in $[6,32,5,43]$ will arise from the type of cells considered, the definition of the control point set $\mathcal{Q}_{c}$ and the way condition (18) is ensured.

Let us remark that, similarly to the 1D case, the first-order scheme (14) provided with the two-states Riemann solver produces entropy at the semi-discrete level.

Remark 4.1. Any semi-discrete scheme based on the one-dimensional two-states Riemann solver (16), and which can be put in the following general form

$$
m_{c} \frac{\mathrm{d} U_{c}}{\mathrm{~d} t}=-\sum_{q \in \mathcal{Q}_{c}} \overline{\mathrm{F}}_{q c} \cdot l_{q c} \boldsymbol{n}_{q c},
$$

ensures an entropy production at the semi-discrete level. Indeed, by means of the Gibbs identity (7), it follows that the semi-discrete scheme (19) produces

$$
\begin{aligned}
m_{c} T_{c} \frac{\mathrm{d} S_{c}}{\mathrm{~d} t} & =m_{c} \frac{\mathrm{d} e_{c}}{\mathrm{~d} t}+\boldsymbol{u}_{c} \cdot m_{c} \frac{\mathrm{d} \boldsymbol{u}_{c}}{\mathrm{~d} t}+p_{c} m_{c} \frac{\mathrm{d} \tau_{c}}{\mathrm{~d} t}, \\
& =\sum_{q \in \mathcal{Q}_{c}} \widetilde{z}_{q c} l_{q c}\left[\left(\overline{\boldsymbol{u}}_{q}-\boldsymbol{u}_{c}\right) \cdot \boldsymbol{n}_{q c}\right]^{2} \geq 0 .
\end{aligned}
$$

The question of the fully discrete entropy production will be addressed in the remainder of the article. Now, another essential property to be ensured in a Lagrangian frame is the accordance with the Geometric Conservation Law (GCL), which means that the new volume computed through the new position of the grid nodes has to be the same as the one derived from the discretization of the governing equation of the specific volume. Through scheme (19) and trajectory equation $\frac{\partial}{\partial t} \boldsymbol{x}_{q}=\overline{\boldsymbol{u}}_{q}$, we know for sure that the GCL will be respected at the semi-discrete level, as soon as the integration of the boundary term in (12) has been carried out exactly, and that the domain control point set $\mathcal{Q}(\omega)=\bigcup_{c} \mathcal{Q}\left(\omega_{c}\right)$ contains all the points required to characterize the grid. This semi-discrete GCL accordance can be rewritten as $\frac{\partial}{\partial t}\left|\omega_{c}\right|=m_{c} \frac{\partial}{\partial t} \tau_{c}$. Now, to get the counterpart of this property at the fully discrete level, i.e. $\left|\omega_{c}\right|=m_{c} \tau_{c}$ at all time, some implicit features have to be added to the time integrator. Indeed, assuming the grid point velocity $\overline{\boldsymbol{u}}_{q}$ is constant during each time step, the 
location of the moving point $\boldsymbol{x}_{q}$, and hence the normals $l_{q c} \boldsymbol{n}_{q c}$, will be linear in time. So, to ensure the discrete GCL, instead of using the normals at time $t^{n}$, one should have taken for instance the normals at time $t^{n}+\Delta t^{n} / 2$, or the half sum of those at time $t^{n}$ and $t^{n+1}$, as it is generally done. However, because no theoretical positivity result holds for implicit time integration, we only use in this article explicit time discretizations. For a first-order in time scheme, the chosen normals are thus those at time level $n$. The GCL will then be ensured only at the semi-discrete level. Consequently, one cannot be certain that $\left|\omega_{c}\right|$, the true geometric volume of cell $\omega_{c}$, yields $\left|\omega_{c}\right|=m_{c} \tau_{c}$. All these considerations are no more relevant in the one-dimensional case, where the normals remain +1 and -1 at all time. For the sake of consistence with the 1D [44], let us define $\left|\widetilde{\omega}_{c}\right|$, such that $\left|\widetilde{\omega}_{c}\right|=m_{c} \tau_{c}$.

In this subsection, a general first-order scheme has been introduced. Particular choices in the type of cells considered, Figure 1, in the definition of the control point set $\mathcal{Q}_{c}$, or in the way the conservation condition (18) is ensured will enable us to recover the schemes presented in $[6,32,5,43]$. To emphasize that, brief specifications of the schemes will now be given. But first, let us remark that the substantial progress made during the past decades on Lagrangian cell-centered schemes have been made possible thanks to the original work of Dukowicz and his co-workers on their CAVEAT code [2], and the seminal work of Loubère et al on high-order numerical schemes applied to the gas dynamics equations in a total Lagrangian frame [1, 29].

$\boldsymbol{G L A C E}$ scheme. In the scheme introduced by Després and Mazeran in [14] and named GLACE in [6] for Godunov-type LAgrangian scheme Conservative for Energy, the moving cells under consideration are assumed to be polygons delimited by straight line edges, as in Figure 1(a). Here, the control point set $\mathcal{Q}_{c}$ identifies with the node set $\mathcal{P}_{c}$. And for any $p \in \mathcal{P}_{c}$, the normal $l_{p c} \boldsymbol{n}_{p c}$ stands for the cell corner normal, such that $l_{p c} \boldsymbol{n}_{p c}=\frac{1}{2}\left(\boldsymbol{x}_{p^{+}}-\boldsymbol{x}_{p^{-}}\right) \times \boldsymbol{e}_{z}$. Finally, to avoid any explicit coupling between the definition of node velocity, a sufficient condition ensuring the scheme conservation, relation (18), writes as follows

$$
\forall p \in \mathcal{P}(\omega), \quad \sum_{c \in \mathcal{C}_{p}} \bar{p}_{p c} l_{p c} \boldsymbol{n}_{p c}=\mathbf{0},
$$

where $\mathcal{P}(\omega)$ stands for the set containing all grid nodes, and $\mathcal{C}_{p}=\mathcal{C}(p)$ for the neighboring cell set of node $p$. This previous relation says that the pressure forces are conserved around the nodes. Substituting solver relation (16) into condition (21) leads to the following equation

$$
\sum_{c \in \mathcal{C}_{p}}[p_{c}^{n} l_{p c} \boldsymbol{n}_{p c}-\underbrace{\widetilde{z}_{p c} l_{p c}\left(\boldsymbol{n}_{p c} \otimes \boldsymbol{n}_{p c}\right)}_{\mathrm{M}_{p c}}\left(\overline{\boldsymbol{u}}_{p}-\boldsymbol{u}_{c}^{n}\right)]=\mathbf{0},
$$

where $\mathrm{M}_{p c}=\widetilde{z}_{p c} l_{p c}\left(\boldsymbol{n}_{p c} \otimes \boldsymbol{n}_{p c}\right)$ is a projection matrix along $\boldsymbol{n}_{p c}$. This last relation allows us to uniquely define the node velocity $\overline{\boldsymbol{u}}_{p}$

$$
\overline{\boldsymbol{u}}_{p}=\left(\sum_{c \in \mathcal{C}_{p}} \mathrm{M}_{p c}\right)^{-1} \sum_{c \in \mathcal{C}_{p}}\left(\mathrm{M}_{p c} \boldsymbol{u}_{c}^{n}+p_{c}^{n} l_{p c} \boldsymbol{n}_{p c}\right) .
$$

EUCCLHYD scheme. A thorough study of the properties of the GLACE node-centered solver reveals a strong sensitivity to cell aspect ratio. Furthermore, the fact that the matrix $M_{p c}$ is a rank-one matrix can lead to severe numerical instabilities due to a too low entropy production, and also makes boundary conditions difficult to implement. In [32], Maire et al proposed an alternative scheme named EUCCLHYD for Explicit Unstructured Cell-Centered Lagrangian HYDrodynamics, 
that successfully solves these problems. This method relies on the same assumptions and design procedure. Thus, similar straight line edged polygonal cells are used, refer to Figure 1(a). The only difference between these two schemes lies in the way the one-dimensional Riemann solver is applied at the grid points. In the GLACE scheme, the solver is applied along the corner normal $l_{p c} \boldsymbol{n}_{p c}$, while in the EUCCLHYD scheme the one-dimensional Riemann solver is applied on each edge surrounding the node. Consequently, the node numerical flux pressure $\bar{p}_{p}$ is not only local to the cell, but also local to the surrounding edges, namely $\bar{p}_{p c}^{-}$and $\bar{p}_{p c}^{+}$. Thus, in cell $\omega_{c}$, one will have two pressures per node, left and right, defined through the one-dimensional two-states solver relation (16). With this particular assumption, the control point set no longer identifies with the node cell set. Here, $\mathcal{Q}_{c}$ is the union of the face control point set $\mathcal{Q}\left(f_{p p^{+}}\right)=\left\{p, p^{+}\right\}$, such that $\mathcal{Q}_{c}=\bigcup_{p \in \mathcal{P}_{c}}\left\{p, p^{+}\right\}$. The

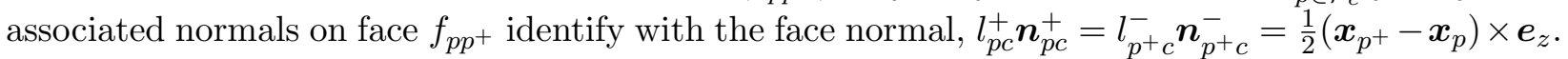
Similar to the GLACE scheme, one recovers the scheme conservation (17) assuming the pressure forces are conserved around the nodes, such that

$$
\forall p \in \mathcal{P}(\omega), \quad \sum_{c \in \mathcal{C}_{p}}\left[\bar{p}_{p c}^{-} l_{p c}^{-} \boldsymbol{n}_{p c}^{-}+\bar{p}_{p c}^{+} l_{p c}^{+} \boldsymbol{n}_{p c}^{+}\right]=\mathbf{0}
$$

which leads to

$$
\sum_{c \in \mathcal{C}_{p}}[p_{c}^{n} l_{p c} \boldsymbol{n}_{p c}-\underbrace{\left(\widetilde{z}_{p c}^{-} l_{p c}^{-}\left(\boldsymbol{n}_{p c}^{-} \otimes \boldsymbol{n}_{p c}^{-}\right)+\widetilde{z}_{p c}^{+} l_{p c}^{+}\left(\boldsymbol{n}_{p c}^{+} \otimes \boldsymbol{n}_{p c}^{+}\right)\right)}_{\mathbf{M}_{p c}}\left(\overline{\boldsymbol{u}}_{p}-\boldsymbol{u}_{c}^{n}\right)]=\mathbf{0},
$$

with $\mathrm{M}_{p c}=\widetilde{z}_{p c}^{-} l_{p c}^{-}\left(\boldsymbol{n}_{p c}^{-} \otimes \boldsymbol{n}_{p c}^{-}\right)+\widetilde{z}_{p c}^{+} l_{p c}^{+}\left(\boldsymbol{n}_{p c}^{+} \otimes \boldsymbol{n}_{p c}^{+}\right)$being the projection matrix along the two corner normals $\boldsymbol{n}_{p c}^{-}$and $\boldsymbol{n}_{p c}^{+}$. This last relation allows us to uniquely define the node velocity $\overline{\boldsymbol{u}}_{p}$, similar to the GLACE scheme, as follows

$$
\overline{\boldsymbol{u}}_{p}=\left(\sum_{c \in \mathcal{C}_{p}} \mathrm{M}_{p c}\right)^{-1} \sum_{c \in \mathcal{C}_{p}}\left(\mathrm{M}_{p c} \boldsymbol{u}_{c}^{n}+p_{c}^{n} l_{p c} \boldsymbol{n}_{p c}\right) .
$$

GLACE extension on conic cells. In [5], Boutin et al introduced an extension of the GLACE scheme on conical mesh, see Figure 1(b). In this configuration, a point $\boldsymbol{x}$ located on the conical face $f_{p p^{+}}$is defined through

$$
\boldsymbol{x}_{\mid p p^{+}}(\zeta)=\frac{(1-\zeta)^{2} \boldsymbol{x}_{p}+2 \nu \zeta(1-\zeta) \boldsymbol{x}_{m}+\zeta^{2} \boldsymbol{x}_{p^{+}}}{(1-\zeta)^{2}+2 \nu \zeta(1-\zeta)+\zeta^{2}},
$$

where $\zeta \in[0,1]$ is the curvilinear abscissa, $m$ the conic control point and $\nu>0$ the associated weight of the conic. For $\nu=0$, one recovers a straight line, while for $\nu=1$ the conic identifies with a Bezier curve. For the sake of conciseness, let us define $\lambda_{p}(\zeta)=(1-\zeta)^{2} / g(\zeta), \lambda_{m}(\zeta)=2 \nu \zeta(1-\zeta)^{2} / g(\zeta)$ and $\lambda_{p^{+}}(\zeta)=\zeta^{2} / g(\zeta)$, with $g(\zeta)=(1-\zeta)^{2}+2 \nu \zeta(1-\zeta)+\zeta^{2}$, so that equation (25) reformulates as

$$
\boldsymbol{x}_{\mid p p^{+}}(\zeta)=\lambda_{p}(\zeta) \boldsymbol{x}_{p}+\lambda_{m}(\zeta) \boldsymbol{x}_{m}+\lambda_{p^{+}}(\zeta) \boldsymbol{x}_{p^{+}}
$$

In the GLACE conical mesh extension, the control point set is defined as the union of the nodes set and the set containing all the conic control points of cell $\omega_{c}$, i.e. $\mathcal{Q}_{c}=\mathcal{P}_{c} \cup \mathcal{M}_{c}$ with $\mathcal{M}_{c}$ being the conic control point set. The associated normals are then respectively defined as

$$
l_{p c} \boldsymbol{n}_{p c}=\int_{p^{-}}^{p} \lambda_{p} \boldsymbol{n} \mathrm{d} s+\int_{p}^{p^{+}} \lambda_{p} \boldsymbol{n} \mathrm{d} s
$$


as well as

$$
l_{m c} \boldsymbol{n}_{m c}=\int_{p^{-}}^{p} \lambda_{m} \boldsymbol{n} \mathrm{d} s
$$

In these definitions, the normal $\boldsymbol{n} \mathrm{d} s$ is computed through the conic parametrization (25) such that $\boldsymbol{n} \mathrm{d} s=\mathrm{d} \boldsymbol{x}_{\mid p p^{+}} \times \boldsymbol{e}_{z}$. Finally, the scheme conservation (17) is enforced assuming that the pressure forces are conserved around the node, and around the conic control point, which can be put into the two following relations

$$
\begin{aligned}
& \forall p \in \mathcal{P}(\omega), \quad \sum_{c \in \mathcal{C}_{p}} \bar{p}_{p c} l_{p c} \boldsymbol{n}_{p c}=\mathbf{0}, \\
& \forall m \in \mathcal{M}(\omega), \quad\left(\bar{p}_{m L}-\bar{p}_{m R}\right) l_{m L} \boldsymbol{n}_{m L}=\mathbf{0},
\end{aligned}
$$

with $\mathcal{M}(\omega)$ the set containing all conic control points in the domain, and for given conic control point $m, \omega_{L}$ and $\omega_{R}$ stand for the neighboring cells sharing the conical face. The first relation (27) gives the definition of the nodes velocity

$$
\overline{\boldsymbol{u}}_{p}=\left(\sum_{c \in \mathcal{C}_{p}} \mathrm{M}_{p c}\right)^{-1} \sum_{c \in \mathcal{C}_{p}}\left(\mathrm{M}_{p c} \boldsymbol{u}_{c}^{n}+p_{c}^{n} l_{p c} \boldsymbol{n}_{p c}\right),
$$

where $\mathrm{M}_{p c}=\widetilde{z}_{p c} l_{p c}\left(\boldsymbol{n}_{p c} \otimes \boldsymbol{n}_{p c}\right)$. However, the problem deriving from relation (28) is not invertible, and only provides a condition on the normal component of $\overline{\boldsymbol{u}}_{m}$ as follows

$$
\overline{\boldsymbol{u}}_{m} \cdot \boldsymbol{n}_{m L}=\left(\frac{\widetilde{z}_{m L} \boldsymbol{u}_{L}^{n}+\widetilde{z}_{m R} \boldsymbol{u}_{R}^{n}}{\widetilde{z}_{m L}+\widetilde{z}_{m R}}\right) \cdot \boldsymbol{n}_{m L}-\frac{p_{R}^{n}-p_{L}^{n}}{\widetilde{z}_{m L}+\widetilde{z}_{m R}} .
$$

The tangential component of $\overline{\boldsymbol{u}}_{m}$ is then evaluated from an upwind point of view as

$$
\overline{\boldsymbol{u}}_{m} \cdot \boldsymbol{t}_{m L}= \begin{cases}\boldsymbol{u}_{L}^{n} \cdot \boldsymbol{t}_{m L}, & \text { if } \overline{\boldsymbol{u}}_{m} \cdot \boldsymbol{n}_{m L}>0, \\ \boldsymbol{u}_{R}^{n} \cdot \boldsymbol{t}_{m L}, & \text { if } \overline{\boldsymbol{u}}_{m} \cdot \boldsymbol{n}_{m L}<0, \\ \left(\frac{\widetilde{z}_{m L} \boldsymbol{u}_{L}^{n}+\widetilde{z}_{m R} \boldsymbol{u}_{R}^{n}}{\widetilde{z}_{m L}+\widetilde{z}_{m R}}\right) \cdot \boldsymbol{t}_{m L}, & \text { if } \overline{\boldsymbol{u}}_{m} \cdot \boldsymbol{n}_{m L}=0,\end{cases}
$$

where $\boldsymbol{t}_{m L}$ stands for a unit vector orthogonal to $\boldsymbol{n}_{m L}$.

LCCDG scheme. Both GLACE and EUCCLHYD schemes have been extended to second-order accuracy. Nonetheless, to go further to third-order and higher, straight line edges geometry can no longer be used. Indeed, it has been proved in [8] that in this case, an implicit linear assumption is made on the fluid flow. Consequently, higher-order curved geometries are required. In [43], we have presented a general high-order cell-centered discretization of the two-dimensional Lagrangian gas dynamics equations, based a discontinuous Galerkin (DG) scheme and high-order curved polygonal cells, that we refer as LCCDG for Lagrangian Cell-Centered Discontinuous Galerkin. We recall here its first-order version, on a $(d+1)^{\text {th }}$ order polygonal cell, see Figure 1(c) for instance for a fourthorder geometry. When we refer to $(d+1)^{\text {th }}$ geometry, we mean that each edge is defined through $(d+1)$ points. The polygonal cell depicted in Figure 1(a) is then referred as a second-order geometry. In [43], the LCCDG schemes developed are based on a total Lagrangian formulation. But in its first-order version, the method is perfectly compatible between the actual and initial configurations. For the sake of simplicity, we recall such scheme on the moving cells. Here, similarly to EUCCLHYD 
schemes, the cell control point set is made of each face control point set, $\mathcal{Q}_{c}=\bigcup_{p \in \mathcal{P}_{c}} \mathcal{Q}\left(f_{p p^{+}}\right)$, where $\mathcal{Q}\left(f_{p p^{+}}\right)$is made of the $(d+1)$ points defining the curvilinear edge which include the nodes $p$ and $p^{+}$. The associated normals on face $f_{p p^{+}}$are then defined as follows

$$
\begin{aligned}
& l_{p c}^{+} \boldsymbol{n}_{p c}^{+}=\int_{0}^{1} \lambda_{p}(\zeta) \sum_{k \in \mathcal{Q}\left(f_{p p^{+}}\right)} \frac{\partial \lambda_{k}}{\partial \zeta}\left(\boldsymbol{x}_{k} \times \boldsymbol{e}_{z}\right) \mathrm{d} \zeta, \\
& l_{p^{+} c^{-} \boldsymbol{n}_{p^{+} c}^{-}}=\int_{0}^{1} \lambda_{p^{+}}(\zeta) \sum_{k \in \mathcal{Q}\left(f_{p p^{+}}\right)} \frac{\partial \lambda_{k}}{\partial \zeta}\left(\boldsymbol{x}_{k} \times \boldsymbol{e}_{z}\right) \mathrm{d} \zeta,
\end{aligned}
$$

as well as, for $q \neq p$ and $q \neq p^{+}$

$$
l_{q c} \boldsymbol{n}_{q c}=\int_{0}^{1} \lambda_{q}(\zeta) \sum_{k \in \mathcal{Q}\left(f_{p p^{+}}\right)} \frac{\partial \lambda_{k}}{\partial \zeta}\left(\boldsymbol{x}_{k} \times \boldsymbol{e}_{z}\right) \mathrm{d} \zeta .
$$

In these definitions, $\lambda_{q}(\zeta)$ denotes the one-dimensional Lagrangian finite element basis functions of degree $d, \zeta \in[0,1]$ the curvilinear abscissa, and $\boldsymbol{x}_{k}$ the actual position of the control point $k \in \mathcal{Q}\left(f_{p p^{+}}\right)$. To recover the scheme conservation, we make use of two complementary sufficient conditions, a pressure force conservation around the grid nodes

$$
\forall p \in \mathcal{P}(\omega), \quad \sum_{c \in \mathcal{C}_{p}}\left[\bar{p}_{p c}^{-} l_{p c}^{-} \boldsymbol{n}_{p c}^{-}+\bar{p}_{p c}^{+} l_{p c}^{+} \boldsymbol{n}_{p c}^{+}\right]=\mathbf{0}
$$

and a pressure force conservation around the face control points

$$
\forall q \in \mathcal{Q}(\omega) \backslash \mathcal{P}(\omega), \quad\left(\bar{p}_{q L}-\bar{p}_{q R}\right) l_{q L} \boldsymbol{n}_{q L}=\mathbf{0},
$$

where $\mathcal{Q}(\omega)$ denotes the set containing all control points defining the curvilinear grid. Condition (30) allows us to define the grid node velocity

$$
\overline{\boldsymbol{u}}_{p}=\left(\sum_{c \in \mathcal{C}_{p}} \mathrm{M}_{p c}\right)^{-1} \sum_{c \in \mathcal{C}_{p}}\left(\mathrm{M}_{p c} \boldsymbol{u}_{c}^{n}+p_{c}^{n} l_{p c} \boldsymbol{n}_{p c}\right),
$$

where $\mathrm{M}_{p c}=\widetilde{z}_{p c}^{-} l_{p c}^{-}\left(\boldsymbol{n}_{p c}^{-} \otimes \boldsymbol{n}_{p c}^{-}\right)+\widetilde{z}_{p c}^{+} l_{p c}^{+}\left(\boldsymbol{n}_{p c}^{+} \otimes \boldsymbol{n}_{p c}^{+}\right)$. Unlike (30), condition (31) does not allow us to fully determine the face control point velocity. But, in light of the Rankine-Hugoniot relations which state that the tangential component of the velocity should be continuous across a discontinuity, see [43] for further details, the face control point velocity is defined as follows

$$
\overline{\boldsymbol{u}}_{q}=\left(\frac{\widetilde{z}_{q L} \boldsymbol{u}_{L}^{n}+\widetilde{z}_{q R} \boldsymbol{u}_{R}^{n}}{\widetilde{z}_{q L}+\widetilde{z}_{q R}}\right)-\frac{p_{R}^{n}-p_{L}^{n}}{\widetilde{z}_{q L}+\widetilde{z}_{q R}} \boldsymbol{n}_{q f_{p p^{+}}} .
$$

\section{Two-dimensional extension of Godunov-type schemes}

Now, similar to the one-dimensional case, [44], to be able to assess the scheme positivity property, we will rewrite the new averaged solution $\mathrm{U}_{c}^{n+1}$ as a convex combination of the previous solution $\mathrm{U}_{c}^{n}$ and some intermediate states. To that end and in the remainder of the article, we will make use extensively of the essential relation

$$
\sum_{q \in \mathcal{Q}_{c}} l_{q c} \boldsymbol{n}_{q c}=\mathbf{0}
$$


which states that the cell control point normals sum to zero. This relation holds for any of the schemes presented previously. Thanks to (34), we are able to rewrite the first-order scheme (14) as

$$
\begin{aligned}
\mathrm{U}_{c}^{n+1} & =\mathrm{U}_{c}^{n}-\frac{\Delta t^{n}}{m_{c}} \sum_{q \in \mathcal{Q}_{c}} \overline{\mathrm{F}}_{q c} \cdot l_{q c} \boldsymbol{n}_{q c}+\frac{\Delta t^{n}}{m_{c}} \mathrm{~F}\left(\mathrm{U}_{c}^{n}\right) \cdot \sum_{q \in \mathcal{Q}_{c}} l_{q c} \boldsymbol{n}_{q c} \\
& =\left(1-\lambda_{c}\right) \mathrm{U}_{c}^{n}+\sum_{q \in \mathcal{Q}_{c}} \lambda_{q c} \overline{\mathrm{U}}_{q c}
\end{aligned}
$$

where $\lambda_{q c}=\frac{\Delta t^{n}}{m_{c}} \widetilde{z}_{q c} l_{q c}$, and $\lambda_{c}=\sum_{q \in \mathcal{Q}_{c}} \lambda_{q c}$. The intermediate state $\overline{\mathrm{U}}_{q c}$ reads

$$
\overline{\mathrm{U}}_{q c}=\mathrm{U}_{c}^{n}-\frac{\left(\overline{\mathrm{F}}_{q c}-\mathrm{F}\left(\mathrm{U}_{c}^{n}\right)\right)}{\widetilde{z}_{q c}} \cdot \boldsymbol{n}_{q c}
$$

which is nothing but the high-dimensional extension of the 1D intermediate states involved in the two-states Riemann solver, see [44]. The \pm sign has simply been replaced by the scalar product with $\boldsymbol{n}_{q c}$. Obviously, to ensure (35) to be a convex combination, the condition $\lambda_{c} \leq 1$ has to be ensured, which provides us with the two-dimensional CFL condition

$$
\Delta t^{n} \leq \sigma_{e} \frac{m_{c}}{\sum_{q \in \mathcal{Q}_{c}} \widetilde{z}_{q c} l_{q c}}
$$

with the CFL coefficient $\sigma_{e}=1$. In the acoustic approximation, where $\widetilde{z}_{q c} \equiv z_{c}^{n}=\rho_{c}^{n} a_{c}^{n}$, this CFL condition reduces to a more classical one

$$
\Delta t^{n} \leq \sigma_{e} \frac{\left|\widetilde{\omega}_{c}^{n}\right|}{a_{c}^{n} \sum_{q \in \mathcal{Q}_{c}} l_{q c}}
$$

where $\left|\widetilde{\omega}_{c}^{n}\right|=m_{c} / \rho_{c}^{n}$ denotes the approximated volume of the cell $\omega_{c}$ at time $t^{n}$ introduced previously. We recall that because the time integration is explicit, the true volumes $\left|\omega_{c}^{n}\right|$ and $\left|\widetilde{\omega}_{c}^{n}\right|$ may differ. However, the differences only arising from the time error, and the problems studied in the Lagrangian framework relying on quite small characteristic computational times, the difference of these two volumes will prove to be extremely small in practice. In $(37), \sum_{q} l_{q c}$ yields different values depending on the scheme being studied. For instance, the CFL condition in the EUCCLHYD scheme will always be smaller than the one in the GLACE scheme. Let us now demonstrate how the discrete general scheme, equation (14), provided with the one-dimensional Riemann solver (16), produces admissible solutions under some constraints to be determined.

\section{First-order positivity-preserving scheme}

Now, similarly to the one-dimensional case, we introduce the convex admissible set we want the numerical solution to remain in

$$
G=\left\{\mathrm{U}=\left(\begin{array}{l}
\tau \\
\boldsymbol{u} \\
e
\end{array}\right), \quad \tau \in\right] \tau_{\min }, \tau_{\max }\left[\text { and } \widehat{\varepsilon}(\mathrm{U})>\varepsilon_{\min }\right\},
$$

where $\widehat{\varepsilon}=\varepsilon-p^{\star} \tau$ in the stiffened EOS case and $\widehat{\varepsilon}=\varepsilon$ otherwise. Thus, assuming $\bigcup_{c}^{n} \in G$, if one is able to prove $\forall q \in \mathcal{Q}_{c}, \overline{\mathrm{U}}_{q c} \in G$ then one can be sure that $\mathrm{U}_{c}^{n+1} \in G$. We will see that 
the one-dimensional analysis done previously can be applied in a straightforward manner to this two-dimensional case. Consequently, the same two different techniques to achieve positivity will be studied, namely the modified Dukowicz solver and the generic wave speed definition provided with an additional time step constraint.

\subsection{Modified Dukowicz solver}

Let us first show how the intermediate states $\overline{\mathrm{U}}_{q c}$ defined in equation (36) can be put into the exact same form as in the one-dimensional case. For instance, regarding the condition $\bar{\tau}_{q c}>\tau_{m i n}$, let us rewrite $\bar{\tau}_{q c}$ as

$$
\begin{aligned}
\bar{\tau}_{q c}-\tau_{\min } & =\tau_{c}^{n}+\frac{\left(\overline{\boldsymbol{u}}_{q}-\boldsymbol{u}_{c}^{n}\right)}{\widetilde{z}_{q c}} \cdot \boldsymbol{n}_{q c}-\tau_{\min } \\
& =\left(\tau_{c}^{n}-\tau_{\min }\right)\left(1+\left(\frac{\tau_{c}^{n}}{\tau_{c}^{n}-\tau_{\min }}\right) \frac{z_{c}^{n}}{\widetilde{z}_{q c}} \frac{\left(\overline{\boldsymbol{u}}_{q}-\boldsymbol{u}_{c}^{n}\right)}{a_{c}^{n}} \cdot \boldsymbol{n}_{q c}\right)
\end{aligned}
$$

which, introducing $v_{q c}$ similarly to the $1 \mathrm{D}$ case

$$
v_{q c}=\frac{\left(\overline{\boldsymbol{u}}_{q}-\boldsymbol{u}_{c}^{n}\right)}{a_{c}^{n}} \cdot \boldsymbol{n}_{q c}
$$

turns into

$$
\bar{\tau}_{q c}-\tau_{\min }=\left(\tau_{c}^{n}-\tau_{\min }\right)\left(1+\left(\frac{\tau_{c}^{n}}{\tau_{c}^{n}-\tau_{\min }}\right) \frac{z_{c}^{n}}{\widetilde{z}_{q c}} v_{q c}\right) .
$$

This is nothing but what has been obtained in the one-dimensional case, see [44]. Same kind of relation can be derived for condition $\bar{\tau}_{q c}<\tau_{\max }$. Now, concerning $\bar{\varepsilon}_{q c}=\bar{e}_{q c}-\frac{1}{2}\left(\overline{\boldsymbol{u}}_{q c}\right)^{2}$, let us first notice that $\overline{\boldsymbol{u}}_{q c} \neq \overline{\boldsymbol{u}}_{q}$. Indeed, $\overline{\boldsymbol{u}}_{q c}$ corresponds directly to one-dimensional Riemann solver at the control point $q$ along the normal $\boldsymbol{n}_{q c}$, while $\overline{\boldsymbol{u}}_{q}$ would correspond to an high-dimensional extension of the one-dimensional solver. That being said, one can easily check that

$$
\overline{\boldsymbol{u}}_{q c}=\left(\overline{\boldsymbol{u}}_{q} \cdot \boldsymbol{n}_{q c}\right) \boldsymbol{n}_{q c}+\left(\boldsymbol{u}_{c}^{n} \cdot \boldsymbol{t}_{q c}\right) \boldsymbol{t}_{q c}
$$

where $\boldsymbol{t}_{q c}$ identifies with the unit tangential vector of cell $\omega_{c}$ at control point $q$, orthogonal to $\boldsymbol{n}_{q c}$. Then, using (43), $\bar{\varepsilon}_{q c}$ can be rewritten as

$$
\widehat{\bar{\varepsilon}}_{q c}-\varepsilon_{\text {min }}=\left(\widehat{\varepsilon}_{c}^{n}-\varepsilon_{\text {min }}\right) A_{q c}+B_{q c},
$$

where the quantity $A_{q c}$ writes

$$
A_{q c}=1-\left(\frac{\widehat{\varepsilon}_{c}^{n}}{\widehat{\varepsilon}_{c}^{n}-\varepsilon_{\min }}\right) \frac{\tau_{c}^{n} \widehat{p}_{c}^{n}}{\widehat{\varepsilon}_{c}^{n}} \frac{z_{c}^{n}}{\widetilde{z}_{q c}} v_{q c}
$$

recalling that $\widehat{p}=p+p_{s}$ for stiffened and $\widehat{p}=p$ otherwise, while $B_{q c}$ is defined as follows

$$
B_{q c}=\frac{1}{2}\left(a_{c}^{n}\right)^{2} v_{q c}^{2}
$$


Again, we have exactly recovered the expressions obtained in [44] in the one-dimensional case. Then, to be able to apply the same analysis as in $1 \mathrm{D}$, let us define the modified Dukowicz wave speed $\widetilde{z}_{q c}$ such as

$$
\widetilde{z}_{q c}=z_{c}^{n}\left(1+\widetilde{\Gamma}\left|v_{q c}\right|\right)
$$

where in the case $\widetilde{\Gamma}=\Gamma$ one recovers the original Dukowicz Hugoniot definition, while for $\widetilde{\Gamma}=\sigma_{v}^{-1}$ one gets the positive modified version of it. Because the same one-dimensional analysis holds, we can state the following proposition.

Proposition 6.1. Any scheme based on the one-dimensional two-states Riemann solver (16) with the particular wave speeds definition (47), and which can be put into the generic form (14) ensures an admissible solution under the CFL condition (37) with $\sigma_{e} \leq 1$ and if

$$
\sigma_{v} \leq \min \left(1-\frac{\tau_{\min }}{\tau_{c}^{n}}, \frac{\tau_{\max }}{\tau_{c}^{n}}-1,\left(1-\frac{\varepsilon_{\min }}{\widehat{\varepsilon}_{c}^{n}}\right) \frac{\rho_{c}^{n} \widehat{\varepsilon}_{c}^{n}}{\widehat{p}_{c}^{n}}\right)
$$

Now, let us address the case of any positive wave speeds definition, $\widetilde{z}_{q c}$, which includes the particular case of the Godunov acoustic solver, but still ensuring the numerical solution admissibility.

\subsection{Generic wave speeds}

To make that possible, similarly to the one-dimensional case, we make use of an additional constraint on the time step permitting the control of the approximated cell volume variation, such as

$$
\left|\frac{\Delta V}{V}\right| \equiv \frac{|| \widetilde{\omega}_{c}^{n+1}|-| \widetilde{\omega}_{c}^{n}||}{\left|\widetilde{\omega}_{c}^{n}\right|}<\sigma_{v},
$$

which reformulates into

$$
\Delta t^{n}<\sigma_{v} \frac{\left|\widetilde{\omega}_{c}^{n}\right|}{\left|\sum_{q \in \mathcal{Q}_{c}} \overline{\boldsymbol{u}}_{q} \cdot l_{q c} \boldsymbol{n}_{q c}\right|} .
$$

Making use of system (14) and recalling that $\Delta V=\Delta t^{n} \sum_{q \in \mathcal{Q}_{c}} \overline{\boldsymbol{u}}_{q} \cdot l_{q c} \boldsymbol{n}_{q c}, \tau_{c}^{n+1}$ rewrites as

$$
\tau_{c}^{n+1}-\tau_{\min }=\left(\tau_{c}^{n}-\tau_{\min }\right)\left(1+\frac{\Delta V}{V}\left(\frac{\tau_{c}^{n}}{\tau_{c}^{n}-\tau_{\min }}\right)\right)
$$

which is perfectly equivalent to what has been obtained in the one-dimensional case [44]. Now, regarding the condition $\widehat{\varepsilon}_{c}^{n+1}>\varepsilon_{\text {min }}$, by means of the first-order scheme (14) and basic manipulations, $\widehat{\varepsilon}_{c}^{n+1}$ can again be split into two terms

$$
\widehat{\varepsilon}_{c}^{n+1}-\varepsilon_{\min }=\left(\widehat{\varepsilon}_{c}^{n}-\varepsilon_{\min }\right) A_{c}+B_{c}
$$

where the quantity $A_{c}$ reads

$$
A_{c}=1-\frac{\Delta V}{V}\left(\frac{\widehat{\varepsilon}_{c}^{n}}{\widehat{\varepsilon}_{c}^{n}-\varepsilon_{\min }}\right) \frac{\tau_{c}^{n} \widehat{p}_{c}^{n}}{\widehat{\varepsilon}_{c}^{n}},
$$


while $B_{c}$ is defined as

$$
B_{c}=\frac{\Delta t^{n}}{m_{c}}\left[\sum_{q \in \mathcal{Q}_{c}} \widetilde{z}_{q c} l_{q c} w_{q c}^{2}-\frac{\Delta t^{n}}{2 m_{c}}\left(\sum_{q \in \mathcal{Q}_{c}} \widetilde{z}_{q c} l_{q c} w_{q c} \boldsymbol{n}_{q c}\right)^{2}\right],
$$

with $w_{q c}=\left(\overline{\boldsymbol{u}}_{q}-\boldsymbol{u}_{c}^{n}\right) \cdot \boldsymbol{n}_{q c}$. Then, if we manage to prove that $B_{c} \geq 0$, it is then sufficient to ensure $A_{c}>0$. Let us first rewrite $B_{c}$ into a matrix-vector form as

$$
B_{c}=\frac{\Delta t^{n}}{m_{c}} \mathrm{M}_{c} \mathrm{~W} \cdot \mathrm{W}
$$

where $\mathrm{W}=\left(w_{1 c}, \ldots, w_{q c}, \ldots, w_{N_{c} c}\right)^{\mathrm{t}}$, with $N_{c}=\left|Q_{c}\right|$ the number of elements contained in $Q_{c}$, and where the generic coefficient $\mathrm{M}_{c}^{q r}$ of matrix $\mathrm{M}_{c}$ reads

$$
\mathrm{M}_{c}^{q r}=\frac{\Delta t^{n}}{m_{c}} \begin{cases}\widetilde{z}_{q c} l_{q c}\left(1-\frac{\Delta t^{n}}{2 m_{c}} \widetilde{z}_{q c} l_{q c}\right), & \text { if } q=r, \\ -\frac{\Delta t^{n}}{2 m_{c}} \widetilde{z}_{q c} \widetilde{z}_{r c} l_{q c} l_{r c}\left(\boldsymbol{n}_{q c} \cdot \boldsymbol{n}_{r c}\right), & \text { if } q \neq r .\end{cases}
$$

Let us recall that if $M_{c}$ is symmetric diagonally dominant with non-negative diagonal entries then $\mathrm{M}_{c}$ is positive semi-definite, see [21] for instance. The matrix $\mathrm{M}_{c}$ yields non-negative diagonal entries if and only if $\Delta t^{n}<\frac{2 m_{c}}{\widetilde{z}_{q c} l_{q c}}$, for any $q \in \mathcal{Q}_{c}$. Now, for the diagonally dominant criterion, it can be proved matrix $\mathrm{M}_{c}$ exhibits such property if and only if

$$
\Delta t^{n} \leq \frac{2 m_{c}}{\sum_{r \in \mathcal{Q}_{c}} \widetilde{z}_{r c} l_{r c}\left|\left(\boldsymbol{n}_{q c} \cdot \boldsymbol{n}_{r c}\right)\right|}
$$

for any $q \in \mathcal{Q}_{c}$, which is always more constraining than the previous condition. Finally, to end up with only one condition per cell, and acknowledging that $\left|\left(\boldsymbol{n}_{q c} \cdot \boldsymbol{n}_{r c}\right)\right| \leq 1$, let us emphasize both conditions, $M_{c}$ is with non-negative diagonal entries and symmetric diagonally dominant, are ensured under the sufficient condition

$$
\Delta t^{n} \leq 2 \frac{m_{c}}{\sum_{q} \widetilde{z}_{q c} l_{q c}}
$$

This condition is nothing but the CFL condition (37) with a CFL coefficient $\sigma_{e}=2$.

Let us note that, similarly to the $1 \mathrm{D}$ case, $B_{c}$ corresponds to the approximation of the time discrete counterpart of the semi-discrete entropy production $T_{c} \frac{\mathrm{d} S_{c}}{\mathrm{~d} t} \geq 0$ of equation (20), $B_{c}$ rewriting as $B_{c}=\varepsilon_{c}^{n+1}-\varepsilon_{c}^{n}-p_{c}^{n}\left(\tau_{c}^{n+1}-\tau_{c}^{n}\right)$. As before, to have $B_{c} \geq 0$ will not ensure the scheme to produce entropy at the discrete level. Actually, one can prove from the concavity of the specific entropy function $S$ that

$$
\frac{1}{T_{c}^{n+1}}\left[\varepsilon_{c}^{n+1}-\varepsilon_{c}^{n}+p_{c}^{n+1}\left(\tau_{c}^{n+1}-\tau_{c}^{n}\right)\right] \leq S\left(\mathrm{U}_{c}^{n+1}\right)-S\left(\mathrm{U}_{c}^{n}\right) \leq \frac{B_{c}}{T_{c}^{n}},
$$

under the assumption that both $\mathrm{U}_{c}^{n}$ and $\mathrm{U}_{c}^{n+1}$ lie in the admissible set $G$. Consequently, to prove any increase in the entropy, one has to determine a time step $\Delta t^{n}>0$ such that the left-hand side of inequality (56) is positive. Such demonstration has been done by Després in [13], in which the solution is assumed to be positive. In the end, even if $B_{c} \geq 0$ will not ensure the scheme to be entropic at the discrete level, it provides us with a sufficient condition for the positivity of the internal energy.

Finally, noticing expressions (50) and (52) are perfectly consistent with what has been obtained in the $1 \mathrm{D}$ case [44], the same analysis holds and is emphasized in the following proposition. 
Proposition 6.2. Any scheme based on the one-dimensional two-states Riemann solver (16) for any positive wave speeds definition, and which can be put into the generic form (14) ensures an admissible solution under the CFL condition (37) with $\sigma_{e} \leq 2$ and the volume variation constraint (49) with

$$
\sigma_{v} \leq \min \left(1-\frac{\tau_{\min }}{\tau_{c}^{n}}, \frac{\tau_{\max }}{\tau_{c}^{n}}-1,\left(1-\frac{\varepsilon_{\min }}{\widehat{\varepsilon}_{c}^{n}}\right) \frac{\rho_{c}^{n} \widehat{\varepsilon}_{c}^{n}}{\widehat{p}_{c}^{n}}\right) .
$$

This proposition holds for any positive wavespeed definition $\widetilde{z}_{q c}>0$, and thus for the particular case of the Godunov acoustic solver.

Summary. Due to the large number of equations introduced, let us summarize the main features of this work presented so far. The problem is to determine conditions for the generic first-order Lagrangian scheme

$$
\mathrm{U}_{c}^{n+1}=\mathrm{U}_{c}^{n}-\frac{\Delta t^{n}}{m_{c}} \sum_{q \in \mathcal{Q}_{c}} \overline{\mathrm{F}}_{q c} \cdot l_{q c} \boldsymbol{n}_{q c}
$$

where $\overline{\mathrm{F}}_{q c}=\left(-\overline{\boldsymbol{u}}_{q}, \mathbb{1}(1) \bar{p}_{q c}, \mathbb{1}(2) \bar{p}_{q c}, \bar{p}_{q c} \overline{\boldsymbol{u}}_{q}\right)^{\mathrm{t}}$ stands for the grid control point numerical flux and is solution of the $1 \mathrm{D}$ two-states Riemann solver

$$
\bar{p}_{q c}=p_{c}^{n}-\widetilde{z}_{q c}\left(\overline{\boldsymbol{u}}_{q}-\boldsymbol{u}_{c}^{n}\right) \cdot \boldsymbol{n}_{q c},
$$

to ensure numerical solutions in the admissible set

$$
G=\left\{\mathrm{U}=(\tau, \boldsymbol{u}, e)^{\mathrm{t}}, \quad \tau \in\right] \tau_{\min }, \tau_{\max }\left[\text { and } \widehat{\varepsilon}(\mathrm{U})>\varepsilon_{\min }\right\} .
$$

To that end, two different techniques are employed. The first one relies on a particular definition of the wavespeeds $\widetilde{z}_{q c}$ depending on $\overline{\boldsymbol{u}}_{q}$, such that

$$
\widetilde{z}_{q c}=\rho_{c}^{n}\left(a_{c}^{n}+\sigma_{v}^{-1}\left|\left(\overline{\boldsymbol{u}}_{q}-\boldsymbol{u}_{c}^{n}\right) \cdot \boldsymbol{n}_{q c}\right|\right) .
$$

Then, a sufficient condition to ensure the numerical solution admissibility is the use of a constant $\sigma_{v} \leq \min \left(1-\frac{\tau_{\min }}{\tau_{c}^{n}}, \frac{\tau_{\max }}{\tau_{c}^{n}}-1,\left(1-\frac{\varepsilon_{\min }}{\widehat{\varepsilon}_{c}^{n}}\right) \frac{\rho_{c}^{n} \widehat{\varepsilon}_{c}^{n}}{\widehat{p}_{c}^{n}}\right)$, and the following CFL condition with $\sigma_{e} \leq 1$

$$
\Delta t^{n} \leq \sigma_{e} \frac{m_{c}}{\sum_{q \in \mathcal{Q}_{c}} \widetilde{z}_{q c} l_{q c}} .
$$

The second technique relaxes this particular definition of the wavespeeds. To that end, in addition to the previous CFL condition with $\sigma_{e} \leq 2$, we make use of a supplementary time step constraint relative to the volume variation as follows

$$
\Delta t^{n}<\sigma_{v} \frac{\left|\widetilde{\omega}_{c}^{n}\right|}{\left|\sum_{q \in \mathcal{Q}_{c}} \overline{\boldsymbol{u}}_{q} \cdot l_{q c} \boldsymbol{n}_{q c}\right|},
$$

where $\left|\widetilde{\omega}_{c}^{n}\right|=m_{c} \tau_{c}^{n}$. Finally, if $\sigma_{v} \leq \min \left(1-\frac{\tau_{\min }}{\tau_{c}^{n}}, \frac{\tau_{\max }}{\tau_{c}^{n}}-1,\left(1-\frac{\varepsilon_{\min }}{\bar{\varepsilon}_{c}^{n}}\right) \frac{\rho_{c}^{n} \widehat{\varepsilon}_{c}^{n}}{\widehat{p}_{c}^{n}}\right)$, the scheme is ensured to produce solutions in $G$. These two techniques involve a constant $\sigma_{v}$ which has proved to be the same. 
Finally, as we did for one dimension in space, we can compare the new time step constraint (49) with the CFL condition (37) in the case of the Dukowicz solver. By means of $\widetilde{z}_{q c}$ definition (47), one can write

$$
\begin{aligned}
\sum_{q \in \mathcal{Q}_{c}} \widetilde{z}_{q c} l_{q c} & >\sum_{q \in \mathcal{Q}_{c}} \rho_{c}^{n} \widetilde{\Gamma}\left|\left(\overline{\boldsymbol{u}}_{q}-\boldsymbol{u}_{c}^{n}\right) \cdot l_{q c} \boldsymbol{n}_{q c}\right|, \\
& >\frac{m_{c}}{\sigma_{v}\left|\widetilde{\omega}_{c}^{n}\right|}\left|\sum_{q \in \mathcal{Q}_{c}} \overline{\boldsymbol{u}}_{q} \cdot l_{q c} \boldsymbol{n}_{q c}\right|
\end{aligned}
$$

And thanks to this last relation, one can state that

$$
\frac{m_{c}}{\sum_{q \in \mathcal{Q}_{c}} \widetilde{z}_{q c} l_{q c}}<\sigma_{v} \frac{\left|\widetilde{\omega}_{c}^{n}\right|}{\left|\sum_{q \in \mathcal{Q}_{c}} \overline{\boldsymbol{u}}_{q} \cdot l_{q c} \boldsymbol{n}_{q c}\right|} .
$$

This second method seems again optimal in term of simplicity and time step, the CFL number $\sigma_{e}$ being twice bigger and the new time step condition (49) being always less constraining than the CFL condition in the Dukowicz or modified Dukowicz solver.

The additional time step restriction technique does not seem limited to the numerical flux used in this paper. A similar procedure can potentially be applied to any other solvers in the Lagrangian framework. It might thus be applied in a straightforward manner to the HLLC Lagrangian scheme presented in [9]. Furthermore, it seems reasonable to say the two positivity-preserving techniques developed here could be generalized to other Lagrangian system of equations, as those involved in the magneto-hydrodynamics or elastic-plastic flow simulation for instance.

We have seen in the one-dimensional case that the high-order extension of the positivity-preserving proof relies directly on the work of Zhang and Shu, [46, 49]. However, in the 2D case, it is no longer possible to apply in a straightforward manner the simple 1D technique, the two-dimensional schemes presented relying on multi-dimensional Riemann solvers used at some control points of the generic polygonal, possibly curved, cells considered. The proof will yet be based on the decomposition of the high-order scheme into a convex combination of first-order schemes.

\section{High-order schemes}

A wide number of different methods can be used to extend the generic first-order formulation (14) to higher-order accuracy, as among others the Monotonic Upstream-Centered Scheme for Conservation Laws (MUSCL) method [25, 26], the essentially non-oscillatory (ENO) finite volume schemes [20, 40], the weighted ENO (WENO) finite volume schemes [28, 22], or the discontinuous Galerkin (DG) method $[11,12]$. The only fundamental assumption is the high-order scheme must satisfy the same equation (14) on the mass averaged values. However, in the definition of the numerical flux $\overline{\mathrm{F}}_{q c}$ through the Riemann solver (16) as well as the respecting definitions of the control point velocity $\overline{\boldsymbol{u}}_{q},(22),(24),(29),(32)$ and (33), the mass average $\mathrm{U}_{c}^{n}$ will be substituted by $\mathrm{U}_{q c}=\mathrm{U}_{h, c}^{n}\left(\boldsymbol{x}_{q}\right)$, the high-order value at point $\boldsymbol{x}_{q}$ within the cells $\omega_{c}$, such that

$$
\bar{p}_{q c}=p_{q c}-\widetilde{z}_{q c}\left(\overline{\boldsymbol{u}}_{q}-\boldsymbol{u}_{q c}\right) \cdot \boldsymbol{n}_{q c} .
$$

Working on the initial configuration, as it is the case in a total Lagrangian formulation, the polynomial $\bigcup_{h, c}^{n}(\boldsymbol{X})$ is defined on cell $\Omega_{c}$, and $\bigcup_{q c}=\bigcup_{h, c}^{n}\left(\boldsymbol{X}_{q}\right)$. Let us highlight that working in a 
total Lagrangian frame, as it is done for instance in $[42,41,43]$, the polynomial $\mathbf{U}_{h, c}^{n}(\boldsymbol{X})$ in the initial configuration may not be a polynomial in the actual configuration $\mathrm{U}_{h, c}^{n}(\boldsymbol{X}(\boldsymbol{x}, t))$. From now on, for sake of conciseness, when not specified, $\boldsymbol{x}$ should be replaced by $\boldsymbol{X}$ in a total Lagrangian frame, as well as $\omega$ by $\Omega$. In both updated and total Lagrangian frames, these polynomials can either be reconstructed from the cell averages of neighboring cells in a finite volume method or evolved in a DG method. To that end, as introduced in the 1D case, one can decide to perform the polynomial reconstruction of the flux variables, as it is done in $[6,33]$. We recall that in this case, the specific volume and total energy remain constant inside the cells, namely $\tau_{h, c}^{n}(x)=\tau_{c}^{n}$ and $e_{h, c}^{n}(x)=e_{c}^{n}$. Because the polynomial reconstruction is performed on the flux variables, and because one only knows the cell averages of the conserved variables, this method is limited to second-order accuracy. To avoid any accuracy discrepancy, one can apply the polynomial reconstruction on the conserved variables. In this case, the pressure is defined pointwisely through the use of the EOS as $p_{h, c}^{n}(\boldsymbol{x})=p\left(\mathbf{U}_{h, c}^{n}(\boldsymbol{x})\right)$. In [9], the authors made use of a second-order ENO method to perform the slope reconstruction, while in [10] a WENO discretization has been used. Obviously, higher order reconstructions may be considered, see for instance [7, 8, 27] for third-order ENO discretizations. Finally, one can also opt for a discontinuous Galerkin discretization. We have previously used such discretization in the total Lagrangian frame to develop generic high-order methods, referred as LCCDG schemes, see [43]. As mentioned before, the first-order version of this scheme is perfectly compatible between the actual and initial configurations. Nonetheless, in the high-order versions, the computation is only performed on the fixed referential configuration, which is assumed to yield straight line edges, even if its image through the fluid flow will admit curved edges, as in Figure 1(c). In this case, the system variables are approximated through polynomial basis functions, for which the corresponding moments are evolved through governing equations. The pressure is then defined pointwisely through the use of the equation of state.

As we did in the one-dimensional case [44], we now introduce relations between the high-order polynomial approximation and the averaged value. For high-order finite volume schemes on the moving configuration, such as those presented in $[6,33,7]$, this relation writes

$$
\mathrm{U}_{c}^{n}=\frac{1}{\left|\omega_{c}\right|} \int_{\omega_{c}} \mathrm{U}_{h, c}^{n}(\boldsymbol{x}) \mathrm{d} v
$$

For the total Lagrangian DG methods introduced in $[41,43]$, this relation writes

$$
\mathrm{U}_{c}^{n}=\frac{1}{m_{c}} \int_{\Omega_{c}} \rho^{0}(\boldsymbol{X}) \mathrm{U}_{h, c}^{n}(\boldsymbol{X}) \mathrm{d} V .
$$

These relations will help us to extend the positivity-preserving proof to the high-orders of accuracy. This proof relies on the fundamental assumption that there exists a two-dimensional quadrature rule on straight line edged polygonal cell, Figure 1(a), exact for polynomial up to degree $K$ for highorder finite volume schemes on moving cell, and $2 K$ for the DG methods on the initial configuration, such that

$$
\int_{\omega_{c}} \phi(\boldsymbol{x}) \mathrm{d} v=\left|\omega_{c}\right| \sum_{\alpha \in \Theta_{c}} w_{\alpha} \phi\left(\boldsymbol{x}_{\alpha}\right),
$$

where $\left\{\left(w_{\alpha}, y_{\alpha}\right)\right\}_{\alpha \in \Theta_{c}}$ are the positive quadrature weights and quadrature points, including the cell control point set, i.e. $\mathcal{Q}_{c} \subset \Theta_{c}$. If no such quadrature is provided by the literature, one can always 
break down the polygonal cells into triangles, and then build the quadrature rule by gathering those on the triangle elements. The triangle quadrature rules developed in [49], deriving from a projection of quadrangle quadrature rules onto triangles, can be used as a starting point. Now, through the use of (61), for the different discretizations presented, the following relation holds

$$
\mathrm{U}_{c}^{n}=\frac{1}{m_{c}} \sum_{\alpha \in \Theta_{c}} m_{\alpha c} \mathrm{U}_{\alpha c}
$$

where, for finite volume schemes on moving cell, $m_{\alpha c}=w_{\alpha} m_{c}$ and $\mathrm{U}_{\alpha c}=\mathrm{U}_{h, c}^{n}\left(\boldsymbol{x}_{\alpha}\right)$, while for the DG scheme on initial cell, $m_{\alpha c}=w_{\alpha} \rho^{0}\left(\boldsymbol{X}_{\alpha}\right)\left|\Omega_{c}\right|$ and $\bigcup_{\alpha c}=\bigcup_{h, c}^{n}\left(\boldsymbol{X}_{\alpha}\right)$. This last expression immediately rewrites

$$
\mathrm{U}_{c}^{n}=\frac{1}{m_{c}} \sum_{\alpha \in \Theta_{c} \backslash \mathcal{Q}_{c}} m_{\alpha c} \mathrm{U}_{\alpha c}+\frac{1}{m_{c}} \sum_{q \in \mathcal{Q}_{c}} m_{q c} \mathrm{U}_{q c}=\frac{m_{c}^{\star}}{m_{c}} \mathrm{U}_{c}^{\star}+\frac{1}{m_{c}} \sum_{q \in \mathcal{Q}_{c}} m_{q c} \mathrm{U}_{q c},
$$

where $m_{c}^{\star}=\sum_{\alpha \in \Theta_{c} \backslash \mathcal{Q}_{c}} m_{\alpha c}$ and $\mathrm{U}_{c}^{\star}=\frac{1}{m_{c}^{\star}} \sum_{\alpha \in \Theta_{c} \backslash \mathcal{Q}_{c}} m_{\alpha c} \mathrm{U}_{\alpha c}$. Now, similarly to what has been done in the $1 \mathrm{D}$ case, we add some artificial fluxes $\mathfrak{F}_{q c}=\left(-\mathfrak{u}_{c}, \mathbb{1}(1) \mathfrak{p}_{q c}, \mathbb{1}(2) \mathfrak{p}_{q c}, \mathfrak{p}_{q c} \mathfrak{u}_{c}\right)^{\mathrm{t}}$, that sum to zero, to the high-order scheme

$$
\mathrm{U}_{c}^{n+1}=\mathrm{U}_{c}^{n}-\frac{\Delta t^{n}}{m_{c}} \sum_{q \in \mathcal{Q}_{c}} \overline{\mathrm{F}}_{q c} \cdot l_{q c} \boldsymbol{n}_{q c}+\frac{\Delta t^{n}}{m_{c}} \sum_{q \in \mathcal{Q}_{c}} \mathfrak{F}_{q c} \cdot l_{q c} \boldsymbol{n}_{q c}
$$

which, along with the use of $\mathbf{U}_{c}^{n}$ decomposition (63), enables us to rewrite $\mathbf{U}_{c}^{n+1}$ as a convex combination

$$
\mathrm{U}_{c}^{n+1}=\frac{m_{c}^{\star}}{m_{c}} \mathrm{U}_{c}^{\star}+\sum_{q \in \mathcal{Q}_{c}} \frac{m_{q c}}{m_{c}} \mathrm{~V}_{q c}
$$

where the $\mathrm{V}_{q c}$ can be expressed as follows

$$
\mathrm{V}_{q c}=\mathrm{U}_{q c}-\frac{\Delta t^{n}}{m_{q c}}\left(\overline{\mathrm{F}}_{q c}-\mathfrak{F}_{q c}\right) \cdot l_{q c} \boldsymbol{n}_{q c} .
$$

For equation (65) to hold, a fundamental assumption on the artificial fluxes has been made, namely they have to sum to zero

$$
\sum_{r \in \mathcal{Q}_{c}} \mathfrak{F}_{r c} \cdot l_{r c} \boldsymbol{n}_{r c}=0
$$

Let us note this condition can be rewritten as

$$
\sum_{r \in \mathcal{Q}_{c} \backslash q} \mathfrak{F}_{r c} \cdot l_{r c} \boldsymbol{n}_{r c}=-\mathfrak{F}_{q c} \cdot l_{q c} \boldsymbol{n}_{q c} .
$$

In the light of (65), assuming $\mathrm{U}_{c}^{\star}$ is in the convex admissible set, if $\forall q \in \mathcal{Q}_{c}, \mathrm{~V}_{q c} \in G$ then $\mathrm{U}_{c}^{n+1}$ is also assured to be admissible. Let us note $\mathrm{U}_{c}^{\star}$ is only made of the contribution of the polynomial solution at time level $n, \mathrm{U}_{h, c}^{n}$, at some quadrature points. So to ensure this quantity to be in $G$, a particular limitation will be designed in Section 9. Now, for $\mathrm{V}_{q c}$, we would like to apply the same analysis as for the first-order scheme. The artificial flux $\mathfrak{F}_{q c}=\left(-\mathfrak{u}_{c}, \mathbb{1}(1) \mathfrak{p}_{q c}, \mathbb{1}(2) \mathfrak{p}_{q c}, \mathfrak{p}_{q c} \mathfrak{u}_{c}\right)^{\mathrm{t}}$, where $\mathfrak{u}_{c}$ stands for some artificial velocity and $\mathfrak{p}_{c}$ some artificial pressure, plays this role. However, 
$\mathrm{V}_{q c}$ defined in (66) does not yet identify with the first-order scheme (14). To that purpose, let us introduce $\overline{\mathfrak{F}}_{r}^{q}$ such that, $\forall r \in \mathcal{Q}_{c}$

$$
\overline{\mathfrak{F}}_{r}^{q}= \begin{cases}\overline{\mathrm{F}}_{q c}, & \text { if } r=q, \\ \mathfrak{F}_{r c}, & \text { otherwise }\end{cases}
$$

where $\overline{\mathfrak{F}}_{r}^{q}=\left(-\overline{\mathfrak{u}}_{r}^{q}, \mathbb{1}(1) \overline{\mathfrak{p}}_{r}^{q}, \mathbb{1}(2) \overline{\mathfrak{p}}_{r}^{q}, \overline{\mathfrak{p}}_{r}^{q} \overline{\mathfrak{u}}_{r}^{q}\right)^{\mathrm{t}}$. In light of (68), definition (66) finally reads

$$
\mathrm{V}_{q c}=\mathrm{U}_{q c}-\frac{\Delta t^{n}}{m_{q c}} \sum_{r \in \mathcal{Q}_{c}} \overline{\mathfrak{F}}_{r}^{q} \cdot l_{r c} \boldsymbol{n}_{r c}
$$

The artificial fluxes $\mathfrak{F}_{r c}$ will be built to ensure that this last expression perfectly mimics the firstorder scheme (14). Substituting in the first-order Riemann solver (16), at the control point $r \in \mathcal{Q}_{c}$, the mean value $\mathrm{U}_{c}^{n}$ with the high-order value $\mathrm{U}_{q c}$, and the numerical flux with the artificial flux tells us that

$$
\begin{aligned}
\sum_{r \in \mathcal{Q}_{c}} \overline{\mathfrak{p}}_{r}^{q} l_{r c} \boldsymbol{n}_{r c} & =\sum_{r \in \mathcal{Q}_{c}}\left(p_{q c}-\widetilde{z}_{r c}^{q}\left(\overline{\mathfrak{u}}_{r}^{q}-\boldsymbol{u}_{q c}\right) \cdot \boldsymbol{n}_{r c}\right) l_{r c} \boldsymbol{n}_{r c}, \\
& =p_{q c} \sum_{r \in \mathcal{Q}_{c}} l_{r c} \boldsymbol{n}_{r c}-\sum_{r \in \mathcal{Q}_{c}} \widetilde{z}_{r c}^{q} l_{r c}\left(\boldsymbol{n}_{r c} \otimes \boldsymbol{n}_{r c}\right)\left(\overline{\mathfrak{u}}_{r}^{q}-\boldsymbol{u}_{q c}\right), \\
& =-\sum_{r \in \mathcal{Q}_{c}} \mathrm{M}_{r c}^{q}\left(\overline{\mathfrak{u}}_{r}^{q}-\boldsymbol{u}_{q c}\right),
\end{aligned}
$$

where $\mathrm{M}_{r c}^{q}=\widetilde{z}_{r c}^{q} l_{r c}\left(\boldsymbol{n}_{r c} \otimes \boldsymbol{n}_{r c}\right)$, and $\widetilde{z}_{r c}^{q}$ may depend on $\overline{\mathfrak{u}}_{r}^{q}$ as in a Dukowicz-like solver. By means of (68), this last relation rewrites

$$
\begin{aligned}
\left(\bar{p}_{q c}-\mathfrak{p}_{q c}\right) l_{q c} \boldsymbol{n}_{q c} & =-\mathrm{M}_{q c}^{q}\left(\overline{\boldsymbol{u}}_{q}-\boldsymbol{u}_{q c}\right)-\left(\sum_{r \in \mathcal{Q}_{c} \backslash q} \mathrm{M}_{r c}^{q}\right)\left(\mathfrak{u}_{c}-\boldsymbol{u}_{q c}\right), \\
& =-\mathrm{M}_{q c}^{q}\left(\overline{\boldsymbol{u}}_{q}-\boldsymbol{u}_{q c}\right)-\mathrm{M}_{c}^{q}\left(\mathfrak{u}_{c}-\boldsymbol{u}_{q c}\right),
\end{aligned}
$$

where $\mathrm{M}_{c}^{q}=\sum_{r \backslash q} \mathrm{M}_{r c}^{q}$. And finally, by means of the high-order Riemann solver relation (58), we are able to define the artificial pressure $\mathfrak{p}_{q c}$ as

$$
\mathfrak{p}_{q c} l_{q c} \boldsymbol{n}_{q c}=p_{q c} l_{q c} \boldsymbol{n}_{q c}+\mathrm{M}_{c}^{q}\left(\mathfrak{u}_{c}-\boldsymbol{u}_{q c}\right) .
$$

In the end, condition (67) will give us the explicit definition of the artificial velocity $\mathfrak{u}_{c}$

$$
\mathfrak{u}_{c}=\left(\sum_{q \in \mathcal{Q}_{c}} \mathrm{M}_{c}^{q}\right)^{-1} \sum_{q \in \mathcal{Q}_{c}}\left[\mathrm{M}_{c}^{q} \boldsymbol{u}_{q c}-p_{q c} l_{q c} \boldsymbol{n}_{q c}\right] .
$$

The artificial flux $\mathfrak{F}_{q c}$ which has been added to the high-order scheme is now fully determined, and can be seen as the high-dimensional extension of the artificial flux used in the 1D case, [44]. Such quantity has allowed us to decompose $\mathrm{U}_{c}^{n+1}$ as a convex combination (65). One can thus state that to have $\mathrm{U}_{c}^{\star}$ and $\forall q \in \mathcal{Q}_{c}, \mathrm{~V}_{q c}$ in the convex admissible set $G$ implies to have the updated numerical solution in average $\mathrm{U}_{c}^{n+1}$ in $G$. Furthermore, in the light of relation (70) as well as the particular definitions of the artificial pressure and velocity, (71) and (72), $\mathrm{V}_{q c}$ identifies perfectly with the first-order scheme (14). Consequently, we can apply the exact same techniques as those presented in the first-order case to ensure $\vee_{q c} \in G$. 


\section{High-order positivity-preserving schemes}

In this section, we focus on the conditions to enforce $\mathrm{V}_{q c} \in G$. Similar to the first-order case, one may choose to use particular non-linear definitions of the local acoustic impedances, or simply an additional time step constraint.

\subsection{Modified Dukowicz solver}

The high-order extension of the modified Dukowicz definitions of $\widetilde{z}_{q c}$, introduced in (47), can be expressed as follows

$$
\widetilde{z}_{q c}=\rho_{q c}\left(a_{q c}+\widetilde{\Gamma}\left|\left(\overline{\boldsymbol{u}}_{q}-\boldsymbol{u}_{q c}\right) \cdot \boldsymbol{n}_{q c}\right|\right)
$$

where $\rho_{q c}, a_{q c}$ and $\boldsymbol{u}_{q c}$ are the high-order values of the density, sound speed and fluid velocity at node $\boldsymbol{x}_{q c}$ within cell $\omega_{c}$, respectively $\boldsymbol{X}_{q c}$ in $\Omega_{c}$ in a total Lagrangian frame. In the case where $\widetilde{\Gamma}=\Gamma$, one recovers the high-order extension of the Dukowicz solver, while for $\widetilde{\Gamma}=\sigma_{v}^{-1}$, equation (73) reads as the extension of the modified one. Given (70) where the artificial numerical flux $\overline{\mathfrak{F}}_{r}^{q}$ has been introduced, we also need to define the local wave speeds relative to this term, $\widetilde{z}_{r c}^{q}$, as

$$
\widetilde{z}_{r c}^{q}=\rho_{q c}\left(a_{q c}+\widetilde{\Gamma}\left|\left(\mathfrak{u}_{c}-\boldsymbol{u}_{q c}\right) \cdot \boldsymbol{n}_{r c}\right|\right),
$$

for $r \neq q$, while for $r=q, \widetilde{z}_{q c}^{q}$ is defined through (73). Each element of equations (70) having been defined explicitly, one may now apply in a straightforward manner the analysis performed on the first-order scheme.

Proposition 8.1. For any high-order discretization presented earlier, assuming $\forall q \in \mathcal{Q}_{c}, \cup_{q c} \in G$ and $\mathrm{U}_{c}^{\star} \in G$, the averaged value $\mathrm{U}_{c}^{n+1}$ is ensured to be admissible provided the numerical fluxes (58) and the particular wave speeds definition (74) with $\widetilde{\Gamma}=\sigma_{v}^{-1}$ under the time step limitation

$$
\Delta t \leq \sigma_{e} \frac{m_{q c}}{\sum_{r \in \mathcal{Q}_{c}} \widetilde{z}_{r c}^{q} l_{r c}},
$$

for all $q \in \mathcal{Q}_{c}$, and with $\sigma_{e} \leq 1$, as well as

$$
\sigma_{v} \leq \min \left(1-\frac{\tau_{\min }}{\tau_{q c}}, \frac{\tau_{\max }}{\tau_{q c}}-1,\left(1-\frac{\varepsilon_{\min }}{\widehat{\varepsilon_{q c}}}\right) \frac{\rho_{q c} \widehat{\varepsilon_{q c}}}{\left|\widehat{p}_{q c}\right|}\right)
$$

Now, if one rather wants to use a simpler solver as the acoustic Godunov one, or any specific definitions of wave speeds as for example $\widetilde{z}_{q c}=\rho_{c}^{n}\left(a_{c}^{n}+\Gamma\left|\left(\overline{\boldsymbol{u}}_{q}-\boldsymbol{u}_{q c}\right) \cdot \boldsymbol{n}_{q c}\right|\right)$, which is generally used in the high-order extension of the Dukowicz solver, one needs to add additional constraints on $\Delta t^{n}$.

\subsection{Generic wave speeds}

In this case, the choice in $\widetilde{z}_{q c}>0$ is free. Thus, one can either use $z_{c}^{n}$ or $\rho_{c}^{n}\left(a_{c}^{n}+\widetilde{\Gamma}\left|\left(\overline{\boldsymbol{u}}_{q}-\boldsymbol{u}_{q c}\right) \cdot \boldsymbol{n}_{q c}\right|\right)$ or even $\rho_{q c}\left(a_{q c}+\widetilde{\Gamma}\left|\left(\overline{\boldsymbol{u}}_{q}-\boldsymbol{u}_{q c}\right) \cdot \boldsymbol{n}_{q c}\right|\right)$ for any $\widetilde{\Gamma}$, or any other definition. The artificial wave speeds $\widetilde{z}_{r c}^{q}$ have to be chosen consistently. Similarly to the first-order case, one can state 
Proposition 8.2. For any high-order discretization presented earlier, assuming $\forall q \in \mathcal{Q}_{c}, \cup_{q c} \in G$ and $\mathrm{U}_{c}^{\star} \in G$, the averaged value $\mathrm{U}_{c}^{n+1}$ is ensured to be admissible provided the numerical fluxes (58) for any positive wave speed definition $\widetilde{z}_{q c}$ under the following time step limitations

$$
\Delta t \leq \sigma_{e} \frac{m_{q c}}{\sum_{r \in \mathcal{Q}_{c}} \widetilde{z}_{r c}^{q} l_{r c}}
$$

for all $q \in \mathcal{Q}_{c}$, and $\sigma_{e} \leq 2$, as well as

$$
\Delta t \leq \sigma_{v} \frac{\tau_{q c} m_{q c}}{\left|\sum_{r \in \mathcal{Q}_{c}} \overline{\mathfrak{u}}_{r}^{q} \cdot l_{r c} \boldsymbol{n}_{r c}\right|}=\sigma_{v} \frac{\tau_{q c} m_{q c}}{\left|\left(\overline{\boldsymbol{u}}_{q}-\mathfrak{u}_{c}\right) \cdot l_{q c} \boldsymbol{n}_{q c}\right|},
$$

for all $q \in \mathcal{Q}_{c}$, where $\sigma_{v}$ has to be such that

$$
\sigma_{v} \leq \min \left(1-\frac{\tau_{\min }}{\tau_{q c}}, \frac{\tau_{\max }}{\tau_{q c}}-1,\left(1-\frac{\varepsilon_{\min }}{\widehat{\varepsilon_{q c}}}\right) \frac{\rho_{q c} \widehat{\varepsilon_{q c}}}{\left|\widehat{p}_{q c}\right|}\right)
$$

The same remark claiming that the second technique is optimal in term of time step still holds in this high-order case. Indeed, in the modified Dukowicz solver case, thanks to definitions (73) and (74), it is quite simple to prove that, $\forall q \in \mathcal{Q}_{c}$

$$
\frac{m_{q c}}{\sum_{r \in \mathcal{Q}_{c}} \widetilde{z}_{r c}^{q} l_{r c}}<\sigma_{v} \frac{\tau_{q c} m_{q c}}{\left|\sum_{r \in \mathcal{Q}_{c}} \overline{\mathfrak{u}}_{r}^{q} \cdot l_{r c} \boldsymbol{n}_{r c}\right|} .
$$

So far, we have proved that assuming $\forall q \in \mathcal{Q}_{c}, \mathrm{U}_{q c}$ and $\mathrm{U}_{c}^{\star}$ lie in the admissible set, there exist a time step ensuring the new numerical solution to be admissible in averaged value. To ensure the required assumptions, we make use of the same kind of limitation introduced in the 1D case, [44].

\section{Positivity-preserving limiter}

In the remainder, $\boldsymbol{x}$ should be replaced by $\boldsymbol{X}$ in a total Lagrangian frame. At time level $n$, the averaged value $\mathrm{U}_{c}^{n}$ is assumed to be in $G$. Then, we modify the polynomial reconstruction $\mathrm{U}_{h, c}^{n}$ to ensure the desired properties, as follows

$$
\widetilde{\mathrm{U}_{h, c}^{n}}(\boldsymbol{x})=\mathrm{U}_{c}^{n}+\theta\left(\mathrm{U}_{h, c}^{n}(\boldsymbol{x})-\mathrm{U}_{c}^{n}\right)
$$

where $\theta \in[0,1]$ is to be determined. The property to be ensured is to yield $\forall q \in \mathcal{Q}_{c}, \widetilde{\mathbf{U}}_{q c} \equiv \widetilde{\mathrm{U}_{h, c}^{n}}\left(\boldsymbol{x}_{q c}\right)$ and $\widetilde{\mathrm{U}}_{c}^{\star} \equiv \frac{1}{m_{c}^{\star}} \sum_{\alpha \in \Theta_{c} \backslash \mathcal{Q}_{c}} m_{\alpha c} \widetilde{\mathrm{U}_{h, c}^{n}}\left(\boldsymbol{x}_{\alpha}\right)$ in the admissible set $G$. To that end, we first enforce the admissibility of the specific volume as follows

$$
\widetilde{\tau_{h, c}^{n}}(\boldsymbol{x})=\tau_{c}^{n}+\theta_{\tau}\left(\tau_{h, c}^{n}-\tau_{c}^{n}\right)
$$

where the coefficient $\theta_{\tau}=\min \left(\theta_{\tau}^{\min }, \theta_{\tau}^{\max }\right)$ is computed such that

$$
\begin{aligned}
& \theta_{\tau}^{\text {min }}=\min \left(1, \frac{\tau_{c}^{n}-\tau_{\min }}{\tau_{c}^{n}-\tau_{m}^{\min }}\right) \quad \text { with } \quad \tau_{m}^{\min }=\min \left(\tau_{c}^{\star}, \min _{q \in \mathcal{Q}_{c}} \tau_{q c}\right), \\
& \theta_{\tau}^{\max }=\min \left(1, \frac{\tau_{\max }-\tau_{c}^{n}}{\tau_{m}^{\max }-\tau_{c}^{n}}\right) \quad \text { with } \quad \tau_{m}^{\max }=\max \left(\tau_{c}^{\star}, \max _{q \in \mathcal{Q}_{c}} \tau_{q c}\right) .
\end{aligned}
$$


Then, for the positivity of the internal energy, the limited polynomial reconstructions of the velocity and total energy are computed through

$$
\begin{aligned}
& \widetilde{\boldsymbol{u}_{h, c}^{n}}(\boldsymbol{x})=\boldsymbol{u}_{c}^{n}+\theta_{\varepsilon}\left(\boldsymbol{u}_{h, c}^{n}-\boldsymbol{u}_{c}^{n}\right), \\
& \widetilde{e_{h, c}^{n}}(\boldsymbol{x})=e_{c}^{n}+\theta_{\varepsilon}\left(e_{h, c}^{n}-e_{c}^{n}\right),
\end{aligned}
$$

where $\theta_{\varepsilon}$ is evaluated in an optimal manner to ensure the required properties. For more details concerning the procedure to get an optimal $\theta_{\varepsilon}$, see the related one-dimensional section in [44].

\section{0. positivity-preserving stability}

We will presently show how the positivity of the numerical scheme yields stability properties. Let us define the piecewise polynomial numerical solution $\bigcup_{h}(\boldsymbol{x}, t)$ defined on $\omega \times[0, T]$ such that

$$
\mathrm{U}_{h}(\boldsymbol{x}, t)=\mathrm{U}_{h, c}^{n}(\boldsymbol{x}), \quad \text { for } \boldsymbol{x} \in \omega_{c} \text { and } t \in\left[t^{n}, t^{n+1}[,\right.
$$

A similar definition can be introduced in the total Lagrangian frame by substituting in (80) $\boldsymbol{x}$ by $\boldsymbol{X}$, and $\omega$ by $\Omega$. We assume the following initialization of the numerical variable mean values

$$
\mathrm{U}_{c}^{0}=\frac{1}{m_{c}} \int_{\Omega_{c}} \rho^{0}(\boldsymbol{X}) U^{0}(\boldsymbol{X}) \mathrm{d} V
$$

where $U^{0}(X)=\left(\frac{1}{\rho^{0}(X)}, \boldsymbol{u}^{0}(X), e^{0}(X)\right)^{\mathrm{t}}$, with $\rho^{0}(X)$ the initial fluid density, $\boldsymbol{u}^{0}(X)$ the initial fluid velocity and $e^{0}(X)$ the initial total energy. Let us introduce the $L_{1}$ and $L_{2}$ norms of a function $\phi$, respectively in the case of finite volume schemes on moving frame

$$
\|\phi\|_{L_{1}}=\frac{m_{c}}{\left|\omega_{c}\right|} \int_{\omega_{c}}|\phi(\boldsymbol{x})| \mathrm{d} v \quad \text { and } \quad\|\phi\|_{L_{2}}=\left(\frac{m_{c}}{\left|\omega_{c}\right|} \int_{\omega_{c}}(\phi(\boldsymbol{x}))^{2} \mathrm{~d} v\right)^{\frac{1}{2}},
$$

and for DG schemes in a total Lagrangian frame

$$
\|\phi\|_{L_{1}}=\int_{\Omega_{c}} \rho^{0}(\boldsymbol{X})|\phi(\boldsymbol{X})| \mathrm{d} V \quad \text { and } \quad\|\phi\|_{L_{2}}=\left(\int_{\Omega_{c}} \rho^{0}(\boldsymbol{X})(\phi(\boldsymbol{X}))^{2} \mathrm{~d} V\right)^{\frac{1}{2}} .
$$

Here, the polynomials $\tau_{h}$ and $\varepsilon_{h}=e_{h}-\frac{1}{2}\left(\boldsymbol{u}_{h}\right)^{2}$ are assumed to be positive everywhere, which is always feasible by limiting enough. Then, setting ourselves in the no external contribution case (17) for sake of simplicity, one can state

$$
\begin{aligned}
& \left\|\tau_{h}\right\|_{L_{1}}=\left\|\frac{1}{\rho^{0}}\right\|_{L_{1}} \quad \text { and } \quad\left\|e_{h}\right\|_{L_{1}}=\left\|e^{0}\right\|_{L_{1}}, \\
& \left\|K_{h}\right\|_{L_{1}}<\left\|e_{h}\right\|_{L_{1}} \quad \text { and } \quad\left\|\boldsymbol{u}_{h}\right\|_{L_{2}}^{2}<m_{\omega}+\left\|e_{h}\right\|_{L_{2}}^{2},
\end{aligned}
$$

where $K=\frac{1}{2} \boldsymbol{u}^{2}$ and $m_{\omega}$ correspond to the kinetic energy and the total mass of domain $\omega$. Let us recall that only the case of the first-order time integration has been tackled so far. In the next section, the high-order time extension will be discussed. 


\section{High-order time discretization}

In Section 7, an high-order space discretization has been employed, while the time integration was carried out by a simple first-order forward Euler method. To reach a global high-order scheme, we make use of Strong Stability Preserving (SSP) Runge-Kutta method, see [40]. See [44] for an example of the used algorithm in the third-order case, for Lagrangian schemes. In light of the fact that these multistage time integration methods write as convex combinations of first-order forward Euler schemes, they will be positivity-preserving as soon as the first-order steps are. Thus, the positive limitation introduced previously, in Section 9, has to be applied at each Runge-Kutta stage. Let us highlight that even though in the first-order time integration case time steps ensuring an admissible numerical solution have been explicitly defined, in the multistage high-order time discretization, one can only be certain that there exists a time step small enough ensuring the global high-order scheme to be positive. Consequently, for the numerical applications we make use of an iterative procedure to determine the time step to be used. Practically, at each time level $n$, we start from an initial time step $\Delta t^{n}$, for instance defined with the first-order restrictions emphasized in Propositions 6.1 and 6.2 with a smaller CFL coefficient as $\sigma_{e}=0.2$. Then, after any Runge-Kutta stage, we assess the average of the new numerical solution to see if it belongs or not to the admissible set $G$. If it is the case, then after having applying the positivity limitation we go forward to the next Runge-Kutta stage. Otherwise, we return to time level $n$ and take $\Delta t^{n} / 2$ as the new time step. By doing this, one does not have to introduce in the implementation the artificial fluxes $\mathfrak{F}_{q c}$ or the related wave speeds $\widetilde{z}_{r c}^{q}$, or any of the quite complex time step restrictions presented. Propositions 8.1 and 8.2 allow us to justify the chosen iterative process admits a positive limit.

\section{Numerical results}

In this numerical results section, similarly to what we did for the 1D [44], we make use of several challenging test cases to demonstrate the performance and robustness of the cell-centered positivitypreserving Lagrangian schemes presented. In the previous successive sections, it has been demonstrated that for the cell-centered Lagrangian schemes considered to be positive, particular definitions of the local acoustic impedance approximation $\widetilde{z}$ have to be used, or an additional constraint on the time step has to be ensured. For the sake of simplicity and computational time, in most of cases presented we make use of the simple Godunov acoustic solver, where $\widetilde{z}=\rho a$. Consequently, if it is not specified, the numerical results displayed are obtained with this acoustic approximation. For the numerical applications, we make use of the first-order and second-order versions of the Lagrangian cell-centered discontinuous Galerkin (LCCDG) schemes presented in [43], for different equations of state. In its first-order version on straight line polygonal mesh, the LCCDG scheme reduces to EUCCLHYD scheme. We will not addressed the third-order case on curved geometries in this paper since the third-order LCCDG scheme still produces some non-physical deformations in the presence of very intense shock waves due to the limitation, see [43]. In the total Lagrangian frame, the whole calculation is performed on the fixed initial grid. However, for a better understanding of the numerical results, the solutions will be displayed on the actual deformed mesh. This is made possible by the knowledge of the deformation gradient tensor during the whole computation.

In this paper, the conditions to ensure the averaged numerical solution to be admissible in the sens $\left.(\tau, \widehat{\varepsilon}) \in] \tau_{\min }, \tau_{\max }[\times] \varepsilon_{\min }, \infty\right]$ have been derived. For practical applications, for ideal and stiffened gas, we make use of $\tau_{\min }=\varepsilon_{\min }=10^{-14}$ and $\tau_{\max }=10^{14}$, while for the detonation product gas

$\tau_{\min }=0.999 \tau_{0}$. Working with the Mie-Grüneisen, we employ $\tau_{\min }=\frac{S_{m}-1}{S_{m}} \tau_{0}$ and $\tau_{\max }=\frac{\tau_{0}}{\eta^{\star}}$. See 
[44] for further details regarding the constants involved in these expressions.

Even if the time step constraints developed in this paper along with the positive limitation introduced previously are enough to ensure the solution to be admissible, an additional limitation might be required in some cases. Indeed, the use of an high-order discontinuous Galerkin discretization leads, in the vicinity of discontinuities, to the apparition of strong spurious oscillations. Obviously, under the constraints derived along this article, these oscillations cannot produce non-admissible solutions. Nonetheless, strong spurious oscillations on the specific volume may lead to a drastic decrease in the time step selection if we want to maintain the admissibility of the numerical solution. To avoid such phenomenon, an additional and more classical limitation may be used. When needed or when we want to improve the global quality of the numerical solution by reducing this spurious oscillation phenomenon, we will turn on the additional limiting procedure. See [44] for further details in the limiting procedure used.

Because the LCCDG have already proved to yield expected accuracy, we refer the interested reader to [43] for a complete convergence analysis on the smooth Taylor-Green vortex test case.

\subsection{The Sedov blast wave problem}

We consider the Sedov problem for a point-blast in a uniform medium. An exact solution based on self-similarity arguments is available, see for instance [23]. The initial density is set to $\rho^{0}=1$, and the fluid at rest as $\boldsymbol{u}^{0}=0$. The pressure is considered to be zero over the domain except at the origin. Thus, we set an initial delta-function energy source at the origin prescribing the pressure in the cell containing the origin as follows, $p_{\text {or }}=(\gamma-1) \rho_{\text {or }} \frac{\varepsilon^{0}}{v_{o r}}$, where $v_{\text {or }}$ denotes the volume of the cell containing the origin and $\varepsilon^{0}$ is the total amount of release energy. By choosing $\varepsilon^{0}=0.244816$, as suggested in [23], the solution consists of a diverging infinite strength shock wave whose front is located at radius $r=1$ at $t=1$, with a peak density reaching 6 . The fluid is modeled by the ideal gas EOS with $\gamma=1.4$. Generally, because one cannot simulate vacuum, the initial pressure is set to $10^{-6}$ over the domain, except at the origin. Here, to make it more challenging, we set the initial pressure to $10^{-14}$.

Now, similarly to what has been done in the $1 \mathrm{D}$ case with the Leblanc shock tube test case, see [44], we assess the resolution of the different solvers, namely the acoustic, Dukowicz and modified Dukowicz solvers, in this point blast problem. Let us point out that, as expected through the analysis presented in this paper, the use of the acoustic solver without the additional time step constraint on the volume variation leads to a crash of the code in this Sedov test case. In this perfect gas problem, consistently with the theory presented, the Dukowicz solver, as well as the modified version of it, will succeed to run until the final time with only the CFL condition enforced. In Figure 3, with the first-order scheme with a $30 \times 30$ Cartesian grid on the domain $(X, Y)=[0,1.2] \times[0,1.2]$, the density maps as well as the density and pressure profiles of the three different solvers are displayed. In the light of Figure 3, regardless the solver used, the numerical solution proves a good agreement with the one-dimensional analytical solution, and the shock wave front is correctly located and almost cylindrical. Further, the density peak almost reaches 6 . However, the overall quality of the final grids obtained with original and modified Dukowicz solvers, Figures 3(b) and 3(c), is superior to the quality of the final grid get in the case of the acoustic solver, see Figure 3(a). Indeed, in Figure 3(a), the grid presents non-convex cells in the 45 degrees direction, while it is not the case on Figures $3(\mathrm{~b})$ and 3(c). Nonetheless, plotting the density and pressure in all cells versus the cell center radius, 


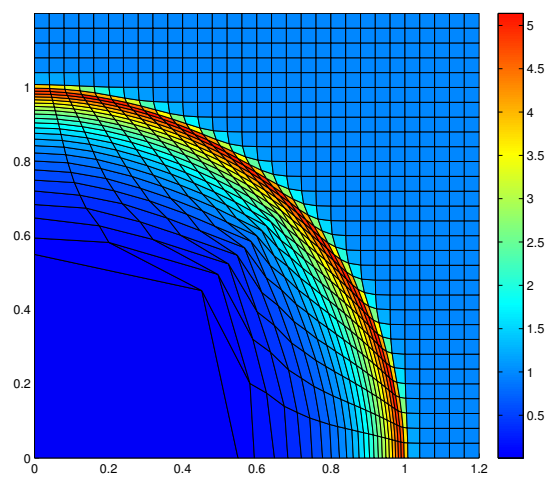

(a) Acoustic solver.

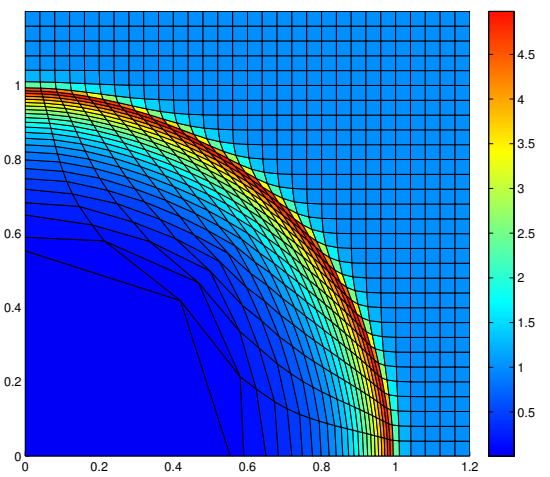

(b) Dukowicz solver.

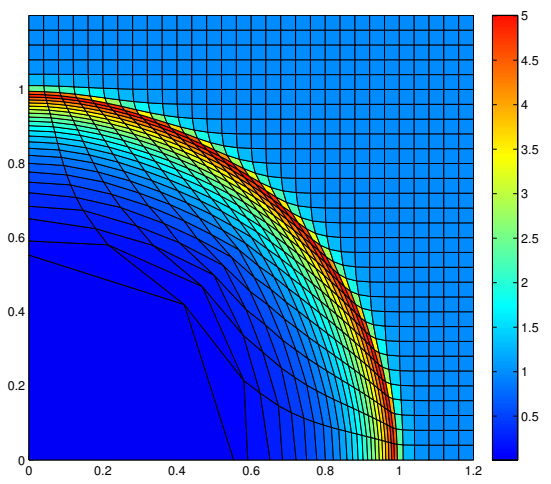

(c) Modified Dukowicz solver.

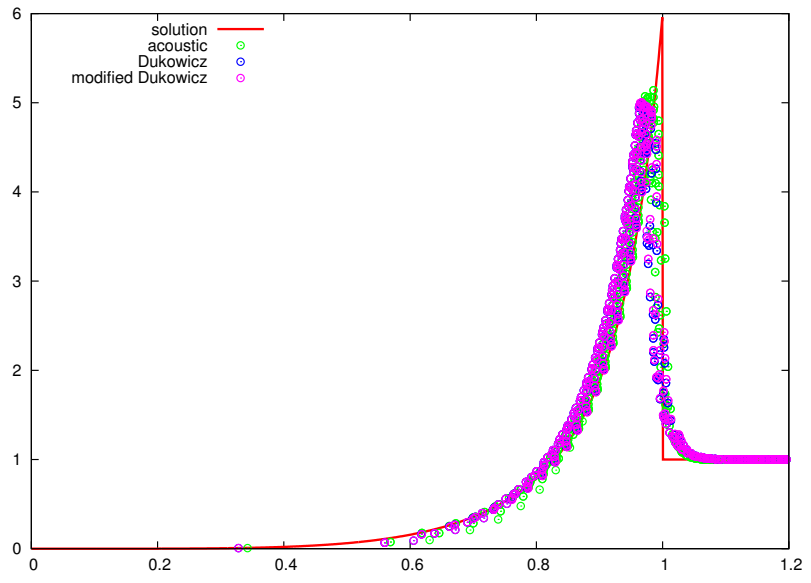

(d) Density profiles.

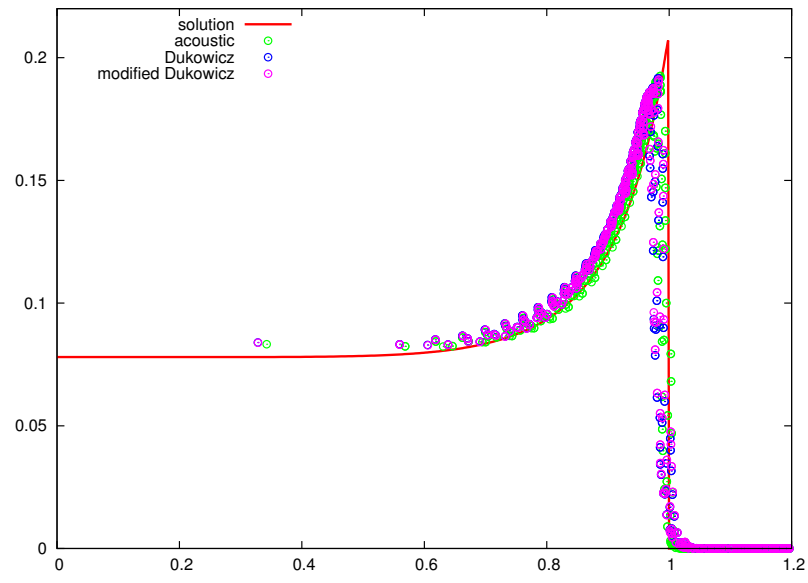

(e) Pressure profiles.

Figure 3: Comparison between solvers on the Sedov problem at time $t=1$ on a $30 \times 30$ Cartesian mesh with first-order schemes.

Figures $3(\mathrm{~d})$ and $3(\mathrm{e})$, one can observe the relative equivalence of the results obtained through the different solvers, even if the acoustic solver seems to be slightly less diffusive. The non-convex cell appearance in the first-order acoustic scheme case is prevented going the second-order. Indeed, the second-order DG scheme provided with the acoustic solver produces a final grid of very good overall quality, see Figure 4(a). Similarly to the 1D cases, the high-order extension dramatically reduces the difference in the results between the different solvers. Finally, we assess the difference in the resolution between the first-order scheme and the limited second-order scheme in this Sedov point blast problem, using the acoustic solver. As expected, given the profiles depicted in Figure 5, and comparing the final meshes obtained, Figures 3(a) and 4(a), it is clear how the high-order scheme captures more accurately the cylindrical and sharp aspect of the front shock.

\subsection{The 123 problem - double rarefaction}

Now, we make use of the two-dimensional extension of the 123 problem we addressed in [44]. The initial domain is defined as $(X, Y) \in[0,4]^{2}$, wherein the fluid is considered perfect with $\gamma=1.4$, the initial density $\rho^{0}=1$ and the initial pressure $p^{0}=0.4$. The fluid velocity is initialized as being outward radial of magnitude 2. Similarly to the 1D case, we compare the final solutions obtained at time $t=1$ with the first-order scheme provided with the three different solvers presented, see 


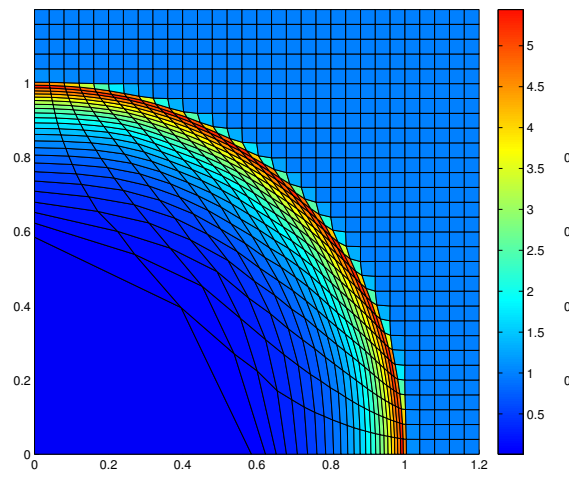

(a) Acoustic solver.

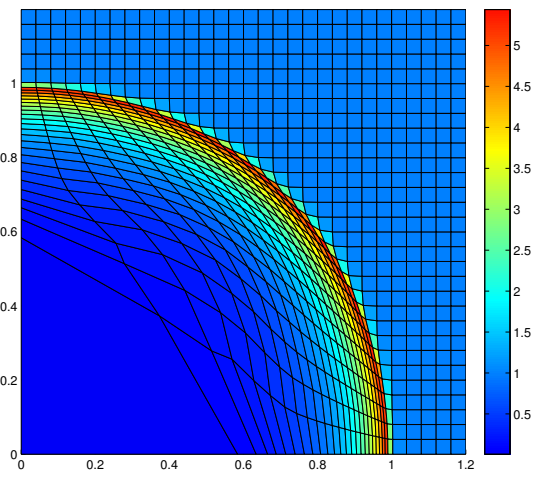

(b) Dukowicz solver.

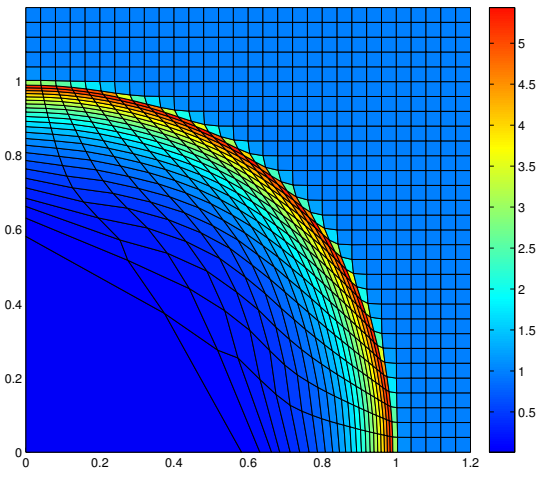

(c) Modified Dukowicz solver.

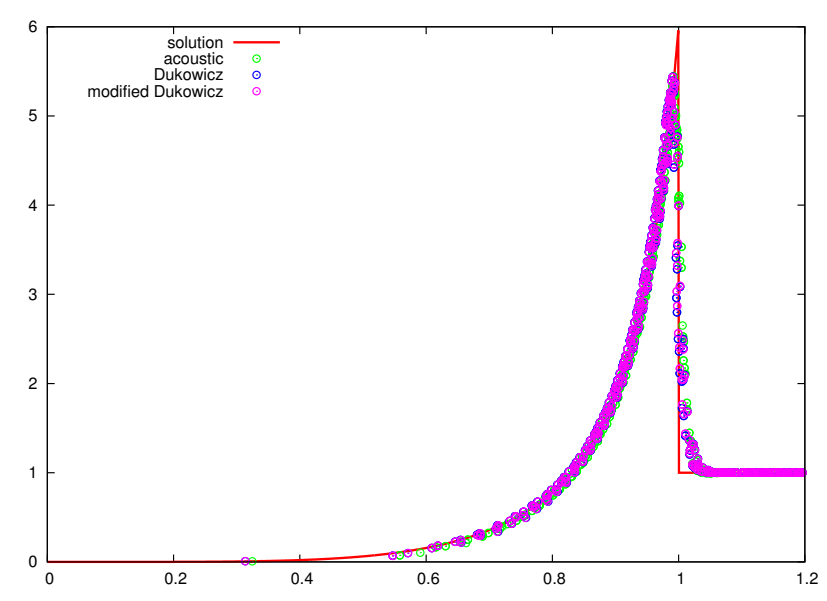

(d) Density profiles.

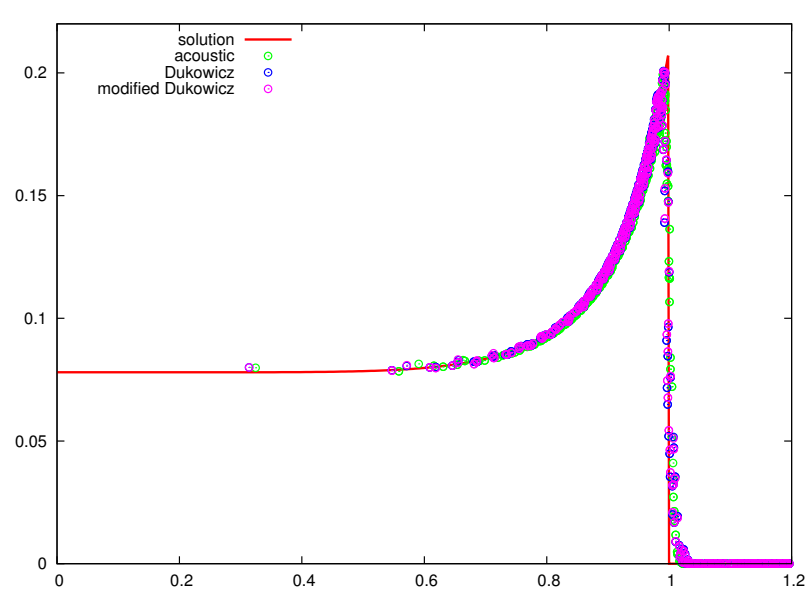

(e) Pressure profiles.

Figure 4: Comparison between solvers on the Sedov problem at time $t=1$ on a $30 \times 30$ Cartesian mesh with limited second-order schemes.

Figure 6(b). Given Figure 6(a), one can clearly see the strong spurious heating phenomenon near the vacuum in this cylindrical rarefaction problem. This strong heating phenomenon is well known, and come from the scheme entropy production even in rarefaction waves, along with the very few number of grid points near the origin in this case. Like in 1D, in the light of Figure 6(b), we can state that in this case the acoustic solver yields the better resolution. In Figure 7, we plot the computation time steps for the three different solvers. As one may have expected, the acoustic solver seems optimal in simplicity and time step in this case. Finally, to observe the benefits of the high-order discretizations, we compare the numerical solutions obtained by means of the first-order scheme and the second-order scheme in this 123 rarefaction problem, using the acoustic solver. As expected, the second-order scheme prove to yield a better resolution than the first-order one, see Figure 8.

\subsection{The Noh problem}

The Noh problem [36] is a well known test case used to validate Lagrangian schemes in the regime of infinite strength shock wave. In this test case, a cold gas modeled by the ideal EOS with $\gamma=5 / 3$ and unit density is given an initial inward radial velocity of magnitude 1. Generally, the initial pressure is given by $p^{0}=10^{-6}$. But, as in the previous Sedov problem, to yield a more challenging 


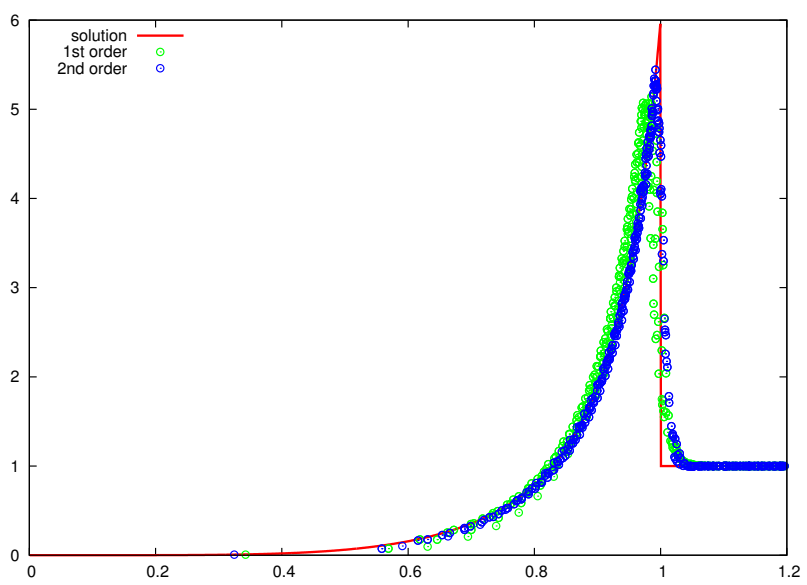

(a) Density profiles.

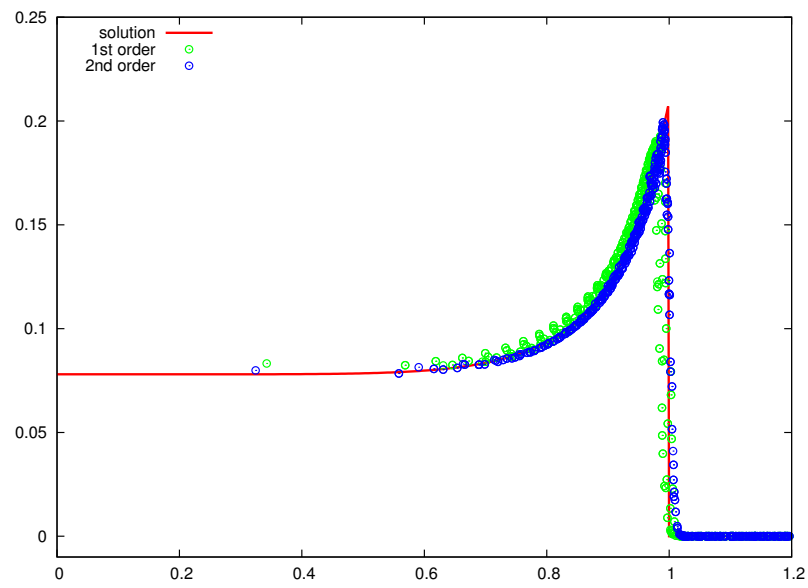

(b) Pressure profiles.

Figure 5: Comparison between first and second-order schemes on the Sedov problem at time $t=1$ on a $30 \times 30$ Cartesian mesh.

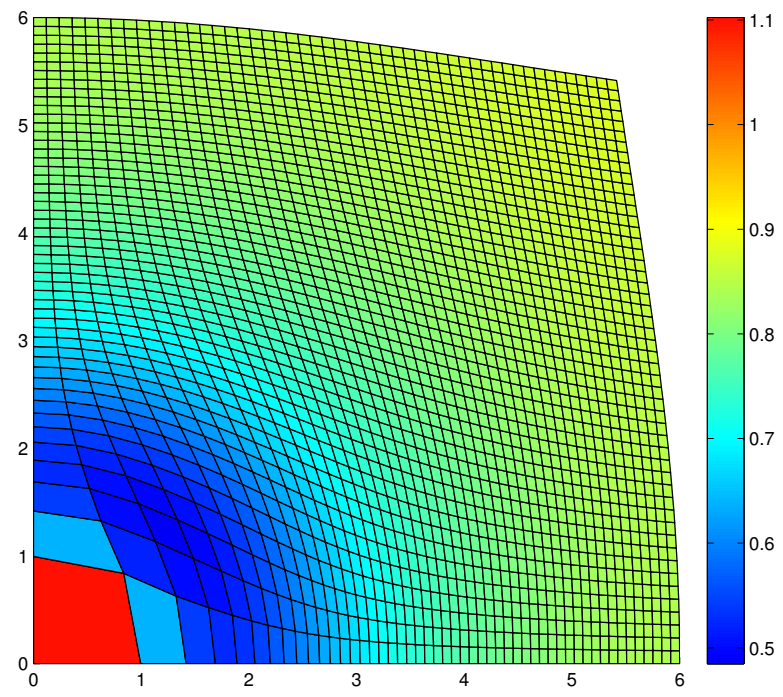

(a) Internal energy map.

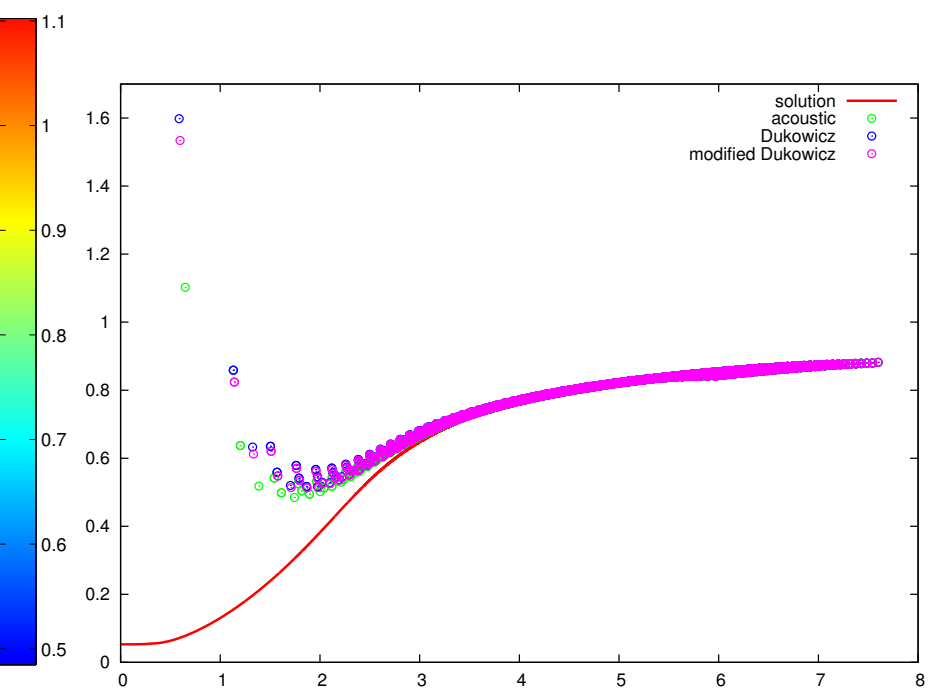

(b) Internal energy profiles

Figure 6: Comparison between solvers on the 123 problem at time $t=1$ on a $50 \times 50$ Cartesian mesh with first-order schemes.

test case we internalize the pressure to $p^{0}=10^{-14}$. A diverging cylindrical shock wave is generated which propagates at speed $D=\frac{1}{3}$. The density plateau behind the shock wave reaches the value 16 . The initial computational domain is defined by $(X, Y)=[0,1] \times[0,1]$. The boundary conditions on the $X$ and $Y$ axis are symmetry conditions whereas a pressure given by $p^{\star}=p^{0}$ is prescribed at $X=Y=1$. We run the Noh problem on a $50 \times 50$ Cartesian grid. This configuration leads to a severe test case since the mesh is not aligned with the flow, and produce some spurious mesh deformations in the 45 degrees direction, see Figure 9(a). This well-known phenomenon can be corrected by using the Dukowicz solver, see Figure 9(b), of the modified Dukowicz one, Figure 9(c). 


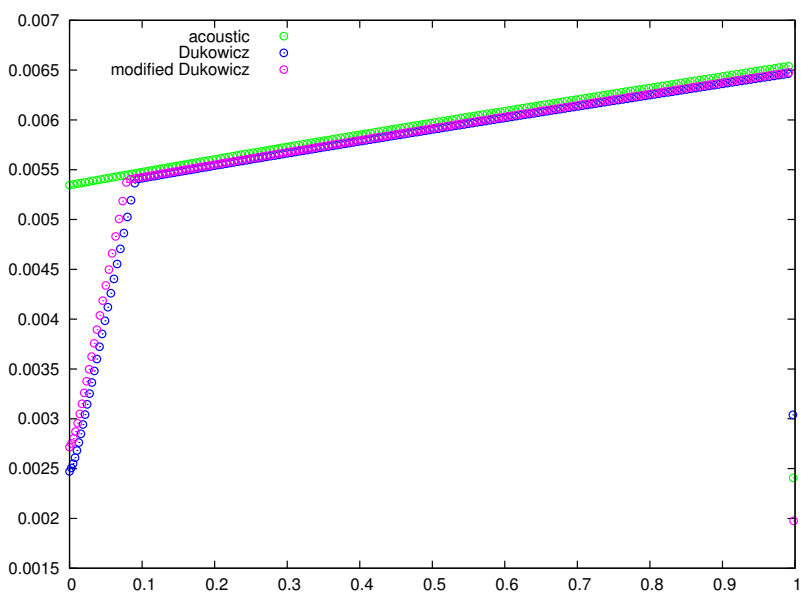

(a) Time steps.

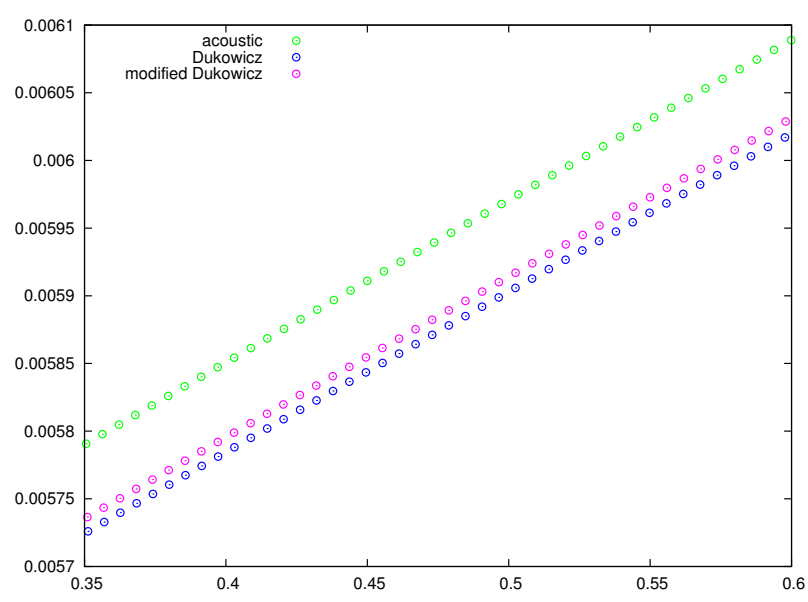

(b) Time steps - zoom.

Figure 7: Time steps of the first-order scheme with different solvers for the 123 problem on a $50 \times 50$ Cartesian mesh.

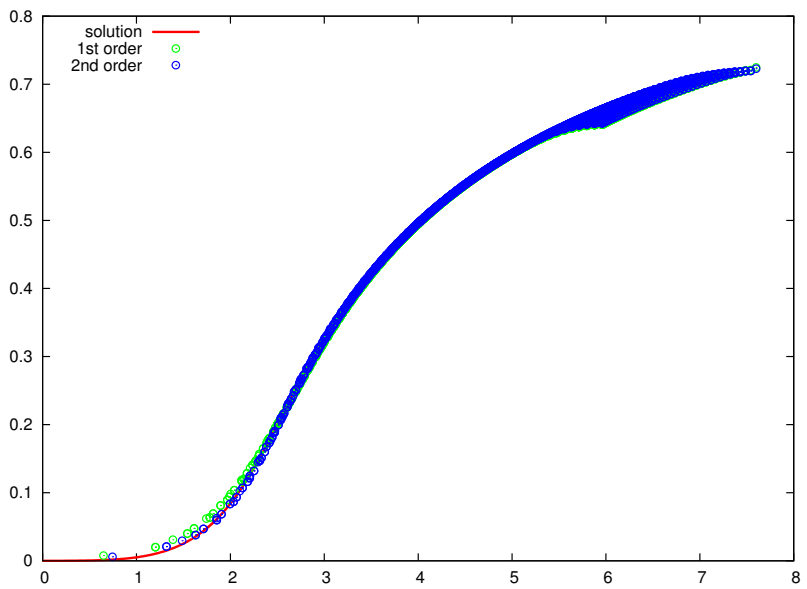

(a) Density profiles.

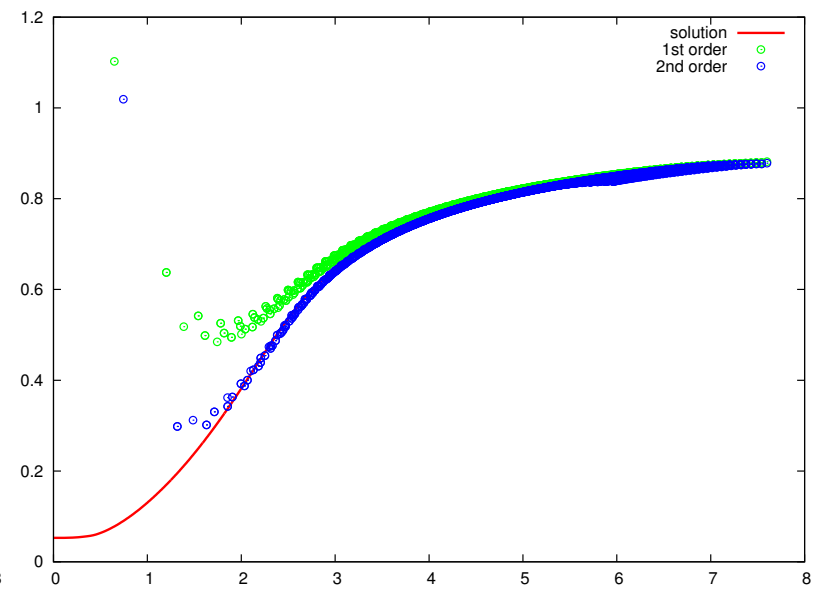

(b) Internal energy profiles.

Figure 8: Comparison between first and second-order schemes on the 123 problem at time $t=1$ on a $50 \times 50$ Cartesian mesh.

Another remedy will be the use of an high-order approximation. We note on Figure 10(a), where the acoustic solver is used, how the limited second-order scheme produces a smooth and cylindrical solution, as well as a shock located at a circle whose radius is approximately 0.2. On Figure 10(b), we observe that the second-order plot is very sharp at the shock wave front and very similar to the one-dimensional cylindrical solution. Moreover the density at the shock plateau is not far from the analytical value. This shows the ability of theses schemes to preserve the radial symmetry of the flow, as well as ensure an admissible numerical solution even in severe test cases as the Noh problem. 


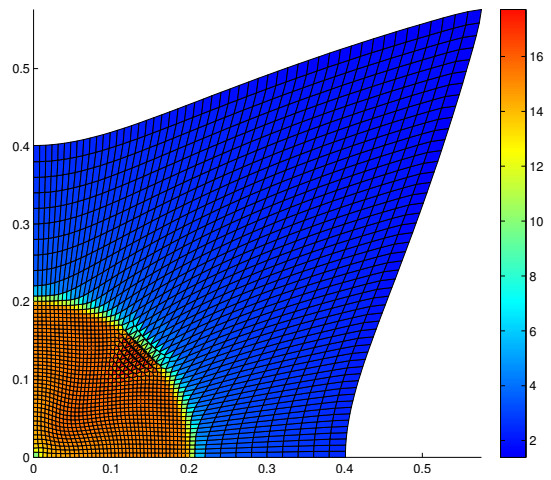

(a) Acoustic solver.

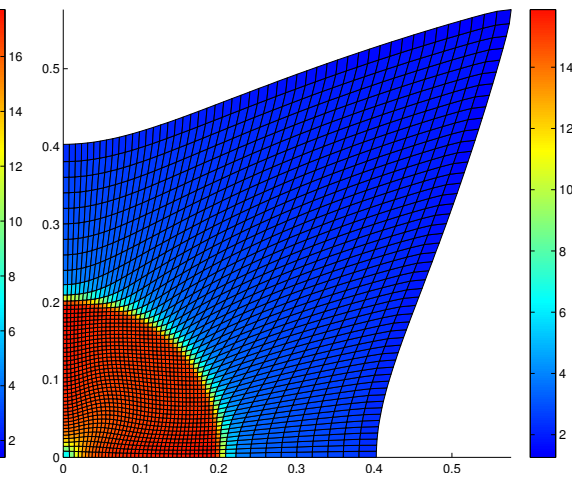

(b) Dukowicz solver.

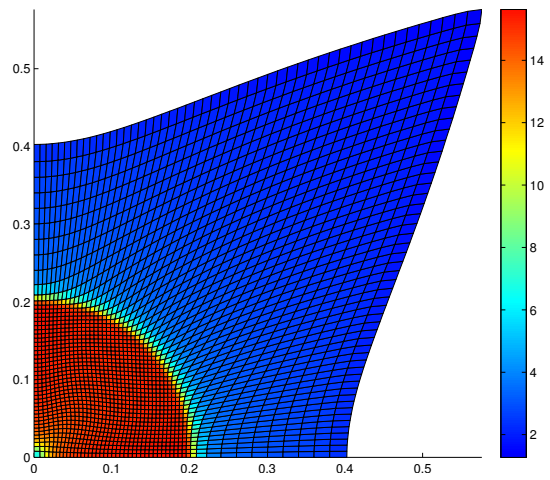

(c) Modified Dukowicz solver.

Figure 9: Comparison between solvers on the Noh problem at time $t=0.6$ on a $50 \times 50$ Cartesian mesh with first-order schemes.

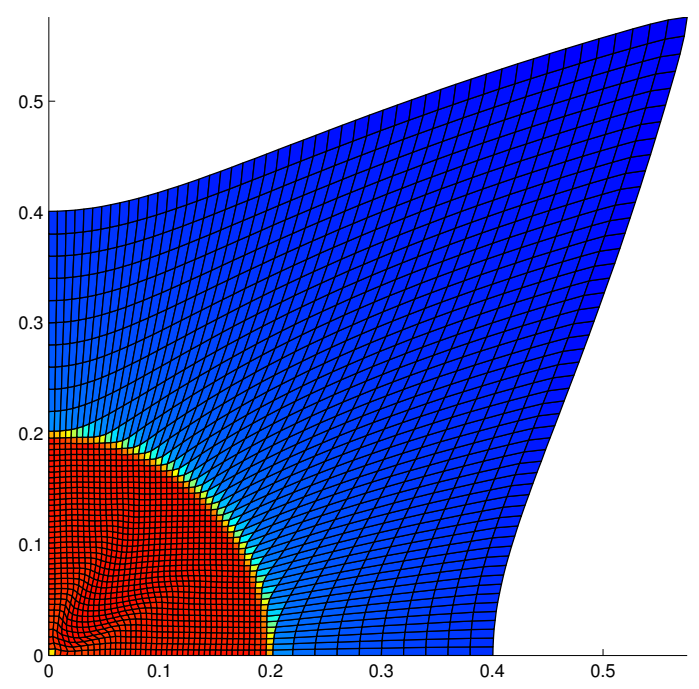

(a) Density map.

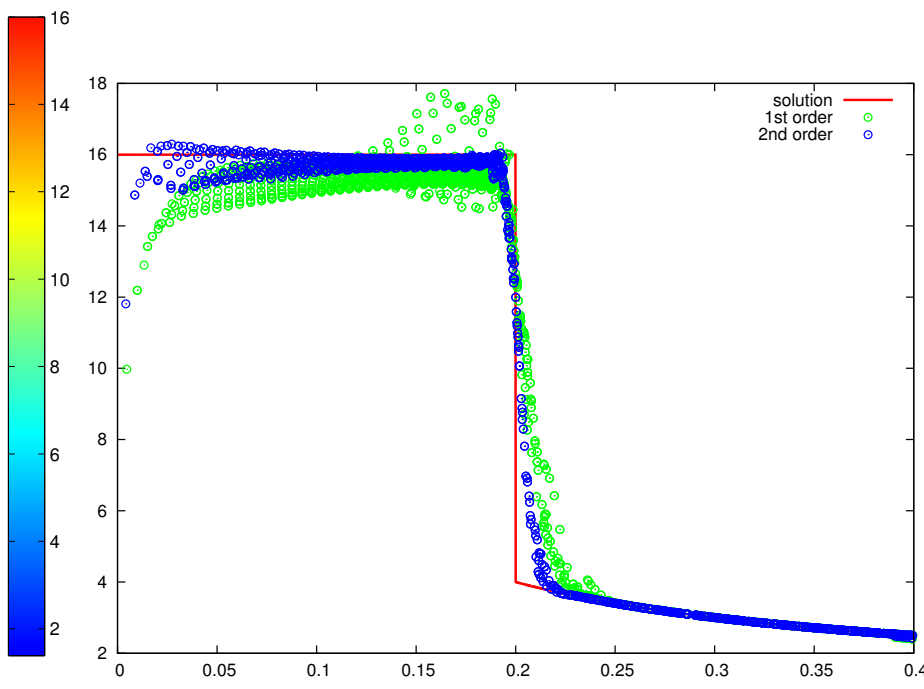

(b) Density profiles.

Figure 10: Comparison between first and second-order schemes on the Noh problem at time $t=0.6$ on a $50 \times 50$ Cartesian mesh.

\subsection{The air-water-air problem}

We make use of the cylindrical two-phase problem presented in [9]. A domain defined in polar coordinates by $(r, \theta) \in[0,1.2] \times[0,2 \pi]$ is considered. The inner and outer parts $(r \in[0,0.2] \bigcup[1.0,1.2])$ of the domain are filled by air, modeled by the ideal EOS with $\gamma=1.4$, while the central part $(r \in[0.2,1.0])$ contains water described through the stiffened gas EOS with $\gamma=7$ and $p_{s}=3000$. The initial data are prescribed as follows

$$
\left(\rho^{0}, \boldsymbol{u}^{0}, p^{0}\right)= \begin{cases}(0.001, \mathbf{0}, 1000), & 0<r<0.2 \\ (1, \mathbf{0}, 1), & 0.2<r<1.0 \\ (0.001 \mathbf{0}, 0.001), & 1.0<r<1.2\end{cases}
$$


Because of the symmetry of the solution, we only simulate a $1 / 8$ part of the full domain, namely $(r, \theta) \in[0,1.2] \times\left[0, \frac{\pi}{4}\right]$. In Figure 11, we display the numerical results obtained with the secondorder DG scheme with the acoustic solver on a $120 \times 9$ polar grid at the final time $t=0.007$. In

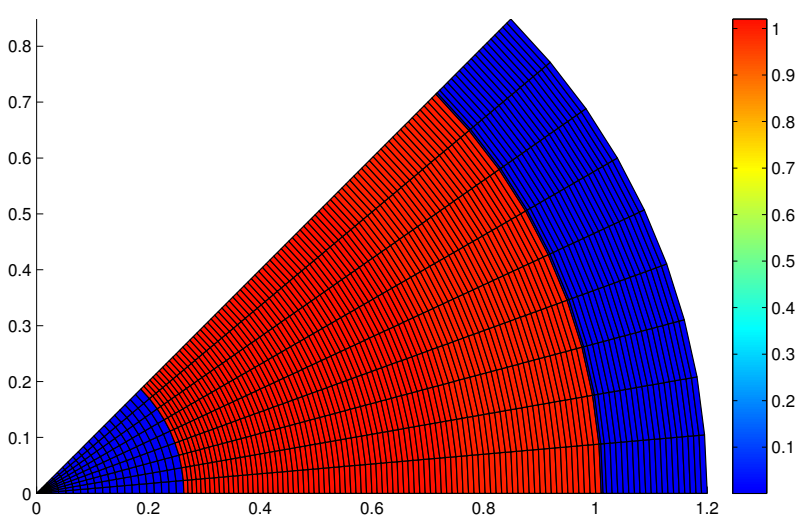

(a) Density map.

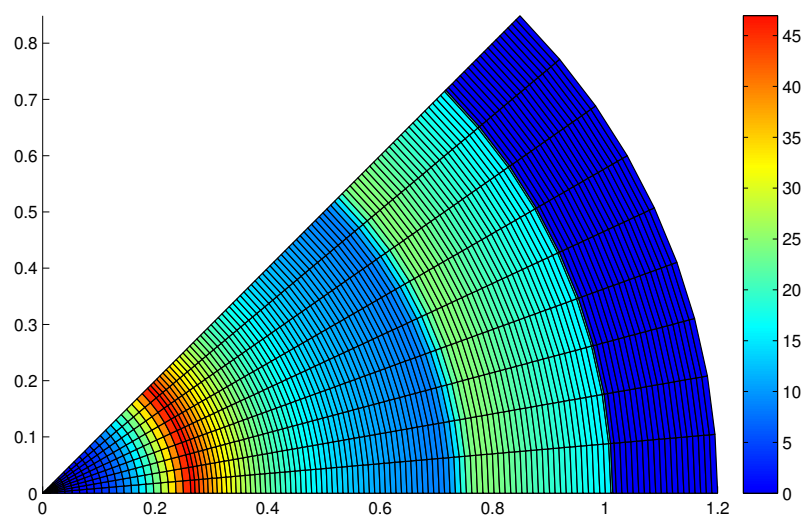

(b) Kinetic energy map.

Figure 11: The air-water-air problem at time $t=0.007$ on the polar domain $[0,1.2] \times\left[0, \frac{\pi}{4}\right]$ made of $120 \times 9$, with the second-order DG scheme.

Figures 11(a) and 11(b), one can clearly that the cylindrical features of the solution are well captured by the numerical scheme. Furthermore, no negative density nor negative internal energy appear during this quite severe problem. In Figures 12(a) and 12(b), the numerical solutions obtained by

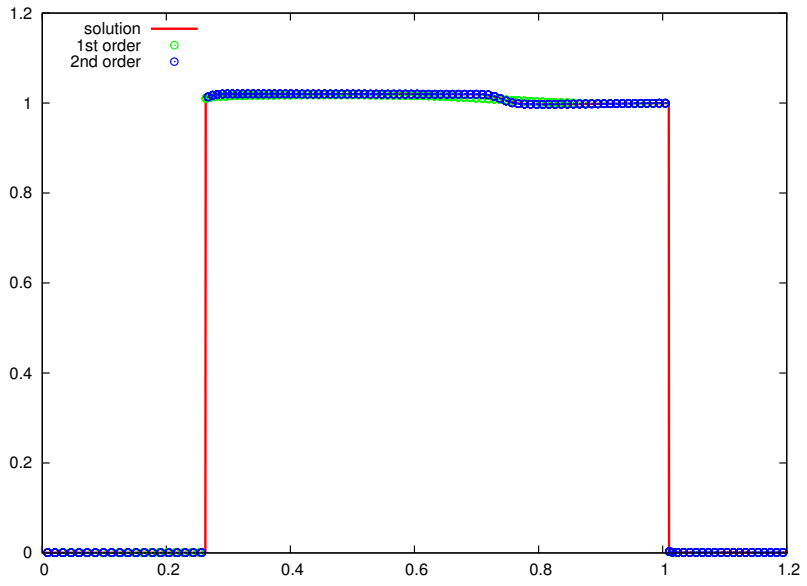

(a) Density profiles.

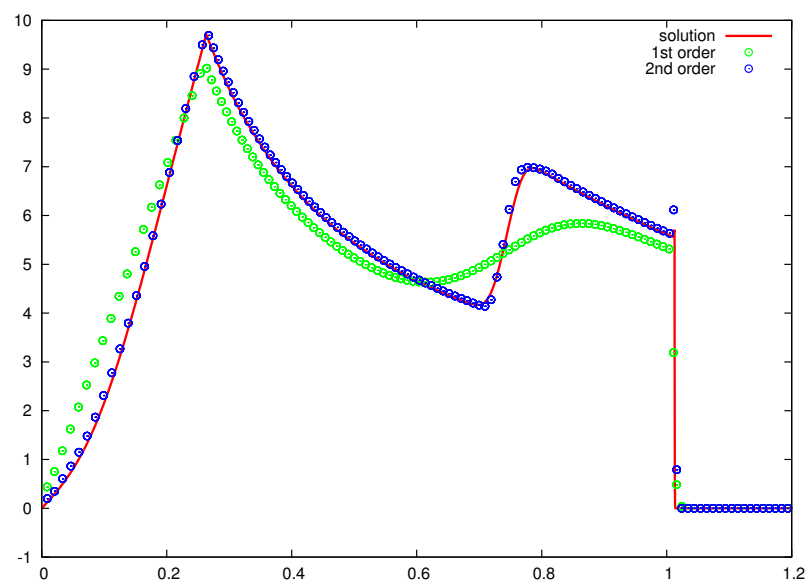

(b) Radial velocity profiles.

Figure 12: Comparison between first and second-order schemes on the air-water-air problem at time $t=0.007$ on the polar domain $[0,1.2] \times\left[0, \frac{\pi}{4}\right]$ made of $120 \times 9$.

means of the first-order and second-order schemes are compared with the reference "exact" solution obtained with $2500 \times 1$ cells on the radial symmetric domain $(r, \theta) \in[0,1.2] \times[0,1]$. In the end, the second-order scheme is shown to have a better resolution. 


\subsection{The underwater TNT explosion}

We now assess, on the polar domain $(r, \theta) \in[0,1.2] \times[0,2 \pi]$, the two-dimensional case of the underwater TNT explosion we have presented in $1 \mathrm{D}$, see [44],. The initial data are prescribed as follows

$$
\left(\rho^{0}, \boldsymbol{u}^{0}, p^{0}\right)= \begin{cases}\left(1.63 \times 10^{-3}, \mathbf{0}, 8.381 \times 10^{3}\right), & 0<r<0.16, \\ \left(1.025 \times 10^{-3}, \mathbf{0}, 1\right), & 0.16<r<1.2 .\end{cases}
$$

The gaseous product of the detonated explosive are initially contained in $r \in[0,0.16]$ and modeled by the JWL EOS with $A_{1}=3.712 \times 10^{5}, A_{2}=3.23 \times 10^{3}, R_{1}=4.15, R_{2}=0.95, \rho_{0}=1.63 \times 10^{-3}$ and $\gamma=1.3$, while the rest of the domain is filled by water described through the stiffened gas EOS with $\gamma=7.15$ and $p_{s}=3.309 \times 10^{2}$. As before, thanks to the symmetry of the solution, we only simulate a $1 / 8$ part of the full domain, namely $(r, \theta) \in[0,1.2] \times\left[0, \frac{\pi}{4}\right]$. In Figure 13, we display the numerical results obtained with the limited second-order DG scheme with the acoustic solver on a $120 \times 9$ polar grid at the final time $t=2.5 \times 10^{-4}$. In Figures $13(\mathrm{a})$ and $13(\mathrm{~b})$, one can clearly see that

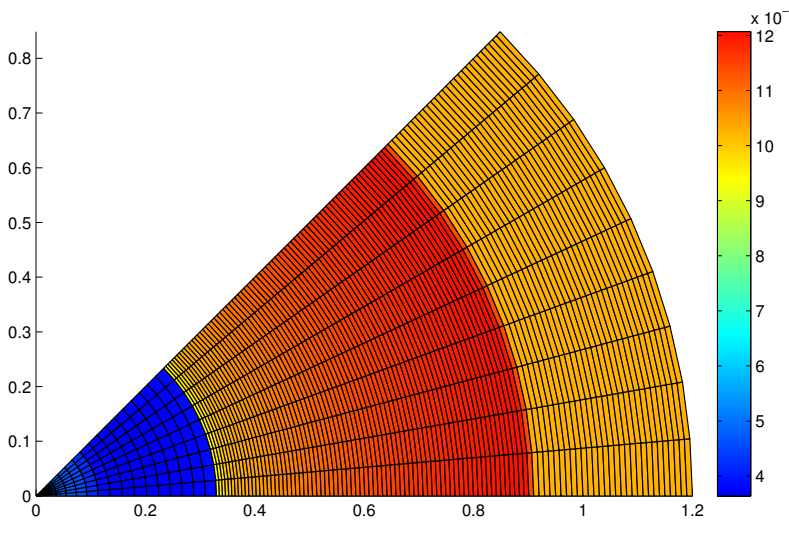

(a) Density map.

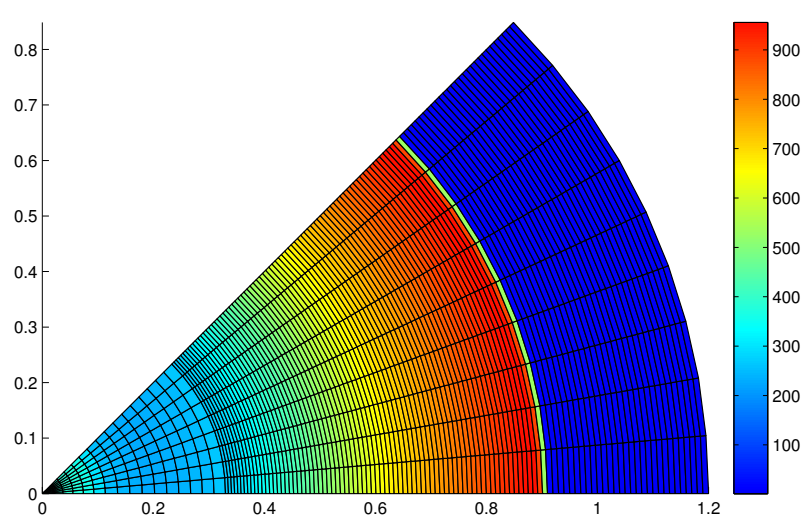

(b) Pressure map.

Figure 13: The spherical underwater TNT charge explosion problem at time $t=2.5 \times 10^{-4}$ on the polar domain $[0,1.2] \times\left[0, \frac{\pi}{4}\right]$ made of $120 \times 9$, with the second-order DG scheme.

the cylindrical features of the solution are well captured by the numerical scheme. Furthermore, no negative density nor negative internal energy appears during this severe multi-material problem. In Figures 14(a) and 14(b), the numerical solutions obtained by means of the first-order and secondorder schemes are compared with the reference "exact" solution obtained with $3000 \times 1$ cells on the radial symmetric domain $(r, \theta) \in[0,1.2] \times[0,1]$. As expected, the second-order scheme proves to have a better resolution. Furthermore, these results are consistent with the ones presented in $[16,9]$.

\subsection{The two-dimensional projectile impact problem}

To end with the numerical results section, we make use of the two-dimensional projectile impact problem introduced and described in [24]. The initial projectile is a rectangular plate of length 5 and height 1 . The material under consideration is aluminum and is thus modeled by the MieGrüneisen equation of state with the following parameters: $\rho_{0}=2785, a_{0}=5328, \Gamma_{0}=2$ and $S_{m}=1.338$. Due the axial symmetry of this problem, we focus in the half problem of the initial domain $(X, Y) \in[0,5] \times[0,0.5]$. The initial velocity is given by $\boldsymbol{u}^{0}=(-150,0)^{\mathrm{t}}$. We take the free 


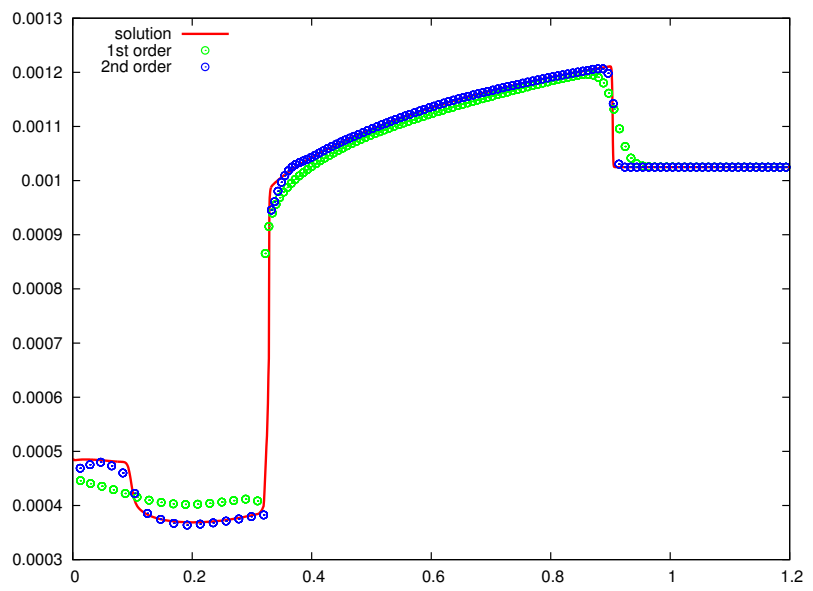

(a) Density profiles.

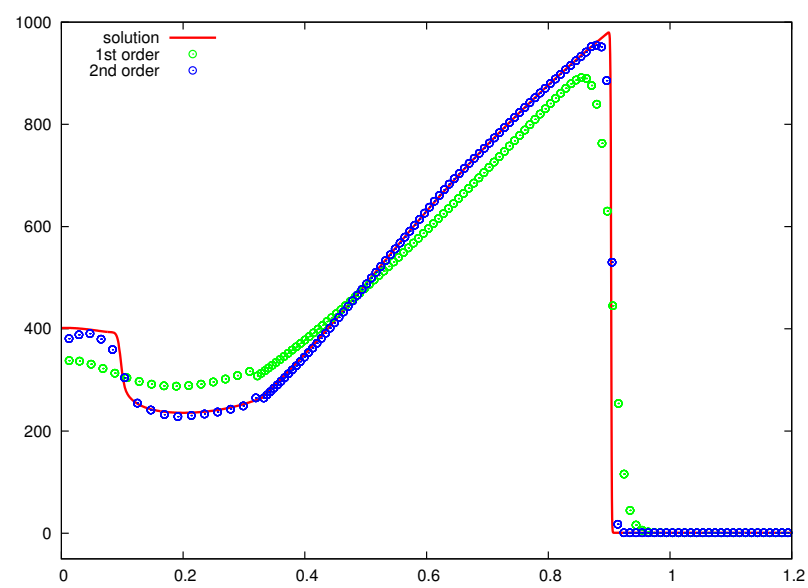

(b) Pressure profiles.

Figure 14: Comparison between first and second-order schemes on the spherical underwater TNT charge explosion problem at time $t=2.5 \times 10^{-4}$ on the polar domain $[0,1.2] \times\left[0, \frac{\pi}{4}\right]$ made of $120 \times 9$.

traction boundary conditions for all the domain boundaries except the left one which is enforced to be a wall boundary. We recall this problem has neither an analytical solution nor experimental results. However, it is a good test to assess the robustness of our numerical method, while keeping in mind that the numerical solution accuracy will be limited by equations model studied, which is in our case the compressible gas dynamics system. In Figure 15, we have displayed the initial grid and the final density maps obtained at time $t=0.005$ by means of the first-order and limited secondorder DG schemes, with the acoustic solver, on a $100 \times 10$ Cartesian grid. Comparing Figures 15(b) and 15(c), we clearly see the second-order final solution grid a lot more deformed than the first-order one. This phenomenon can be explained by the large amount of numerical diffusion inherent of the first-order scheme. As it has been demonstrated in [43] in the case of a Gresho-like vortex problem, in some extreme cases the first-order scheme is unable to simulate appropriately the problems to the final time due to the large numerical diffusion. But obviously, resolving the compressible gas dynamics equations, the numerical schemes presented are not able to capture elastic waves and features. This is the reason why the second-order results, Figure 15(c), are quite far from the ones obtained in [31] with an elastic-plastic second-order cell-centered Lagrangian scheme. Nevertheless, this test case permitted us once more to prove the robustness of the cell-centered Lagrangian schemes presented, as no negative density or negative internal energy appears during the calculation.

\section{Conclusion}

The aim of this paper is to determine different conditions and constraints under which a wide class of cell-centered Lagrangian schemes solving the two-dimensional compressible gas dynamics equations would be positivity-preserving, and thus be assured to produce admissible solution. This article follows the one concerned with the $1 \mathrm{D}$ case, [44]. In this two-dimensional framework, the analysis has been performed on a general first-order cell-centered finite volume formulation based on polygonal meshes defined either by straight line, conical, or any high-order curvilinear edges. Such formulation covers the numerical methods introduced in $[6,32,5,41,43]$. Basically, this positivity-preserving property relies on two different techniques: either a particular definition of the local approximation 


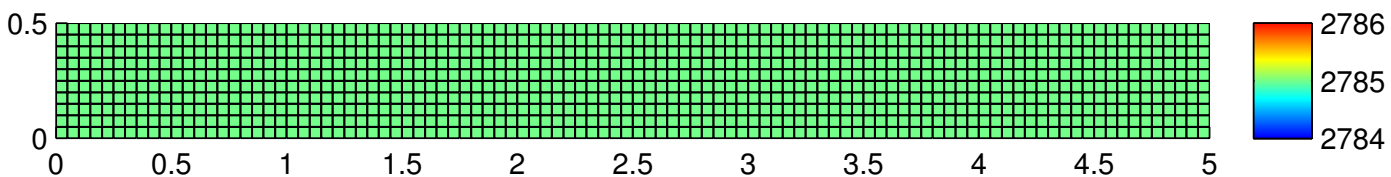

(a) Initial density map.

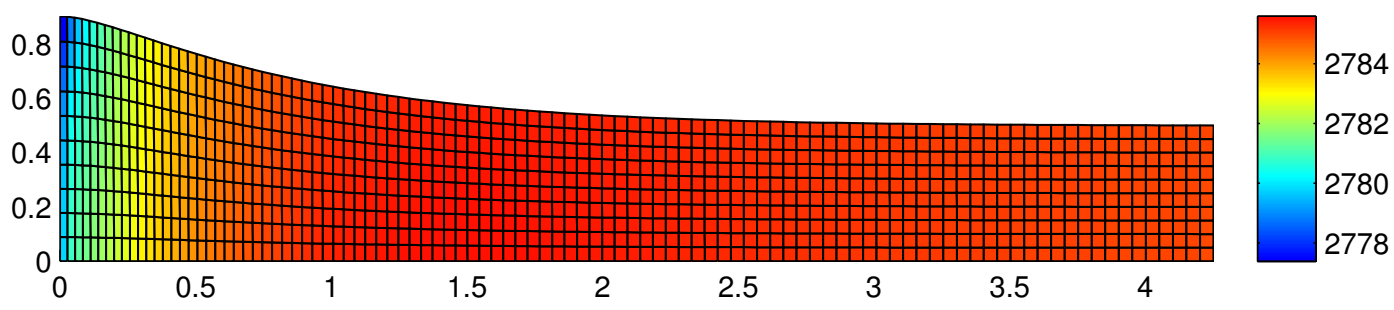

(b) First-order density map.

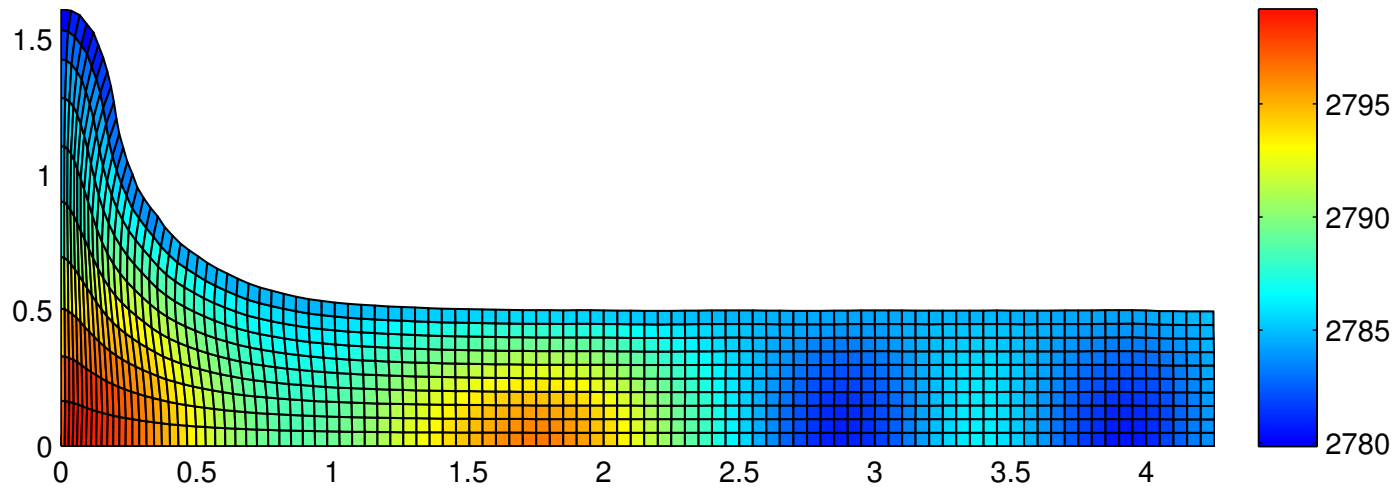

(c) Second-order density map.

Figure 15: Two-dimensional projectile impact problem at time $t=0.005$ on a $100 \times 10$ Cartesian grid.

of the acoustic impedances arising from the approximate Riemann solver, or an additional time step constraint relative to the cell volume variation. The first-order proofs have been extended to the high-orders of accuracy by adapting, to the frame of curvilinear grid and multi-dimensional nodal solvers, the positivity-preserving theory developed in $[45,46,49]$. This work has addressed both ideal and non-ideal equations of state. A wide number of challenging test cases have been used to depict the good performance and robustness of the Lagrangian schemes presented. Let us emphasize that this work is of crucial significance not only for Lagrangian schemes, but also for any methods relying on a purely Lagrangian step, as ALE methods or non-direct Euler schemes based on a Lagrangian step plus a projection.

In the future, we intend to improve the third-order limiting procedure, in the particular case of moving curved geometries, to remedy the appearance of spurious grid deformation found in [43]. This will enable us to demonstrate once more the relevance of the theory developed in this paper, even in the case of third-order scheme based on curving geometries. Then, the whole positivitypreserving theory developed here, as well as the generic discretization introduced for the twodimensional gas dynamics equations, will be generalized to the 3D case, as it is done for instance in $[34,6]$. We also plan to adapt the positive Lagrangian schemes presented here to the Eulerian frame, by adapting the Lagrangian multi-dimensional solvers to the Euler system of equations. Finally, we 
also have in mind to develop a positive DG discretization of the gas dynamics equations written under the updated Lagrangian formulation and to extend its capability to the non-linear elasticity equations.

\section{Acknowledgment}

Research is partially supported by NASA grant NNX12AJ62A and NSF grant DMS-1418750.

\section{References}

[1] R. Abgrall, R. Loubère, and J. Ovadia. A Lagrangian Discontinuous Galerkin-type method on unstructured meshes to solve hydrodynamics problems. Int. J. Numer. Meth. Fluids, 44:645$663,2004$.

[2] F. L. Adessio, J R Baumgardner, J. K. Dukowicz, N. L. Johnson, B. A. Kashiwa, R. M. Rauenzahn, and C. Zemach. CAVEAT: a computer code for fluid dynamics problems with large distortion and internal slip. Technical Report LA-10613-MS, Rev. 1, UC-905, Los Alamos National Laboratory, 1992.

[3] P. Batten, N. Clarke, C. Lambert, and Causon. On the choice of wavespeeds for the HLLC Riemann solver. SIAM J. Sci. Comput., 18:1553-1570, 1997.

[4] C. Berthon, B. Dubroca, and A. Sangam. A local entropy minimum principle for deriving entropy preserving schemes. SIAM J. Numer. Anal., 50(2):468-491, 2012.

[5] B. Boutin, E. Deriaz, P. Hoch, and P. Navaro. Extension of ALE methodology to unstructured conical meshes. ESAIM: Proceedings, 32:31-55, 2011.

[6] G. Carré, S. Delpino, B. Després, and E. Labourasse. A cell-centered Lagrangian hydrodynamics scheme in arbitrary dimension. J. Comp. Phys., 228:5160-5183, 2009.

[7] J. Cheng and C.-W. Shu. A high order ENO conservative Lagrangian type scheme for the compressible Euler equations. J. Comp. Phys., 227(2):1567-1596, 2007.

[8] J. Cheng and C.-W. Shu. A third-order conservative Lagrangian type scheme on curvilinear meshes for the compressible Euler equations. Commun. Comput. Phys., 4:1008-1024, 2008.

[9] J. Cheng and C.-W. Shu. Positivity-preserving Lagrangian scheme for multi-material compressible flow. J. Comp. Phys., 257:143-168, 2014.

[10] J. Cheng and C.-W. Shu. Second order symmetry-preserving conservative Lagrangian scheme for compressible Euler equations in two-dimensional cylindrical coordinates. J. Comp. Phys., 272:245-265, 2014.

[11] B. Cockburn, S.-Y. Lin, and C.-W. Shu. TVB Runge-Kutta local projection discontinuous Galerkin finite element method for conservation laws III: One-dimensional systems. J. Comp. Phys., 84:90-113, 1989.

[12] B. Cockburn and C.-W. Shu. The Runge-Kutta discontinuous Galerkin method for conservation laws V: Multidimensional systems. J. Comp. Phys., 141:199-224, 1998. 
[13] B. Després. Lois de Conservation Euleriennes, Lagrangiennes et méthodes numériques. Mathématiques et Applications. Springer, 2010.

[14] B. Després and C. Mazeran. Lagrangian Gas Dynamics in Two Dimensions and Lagrangian systems. Arch. Rational Mech. Anal., 178:327-372, 2005.

[15] B. Einfeldt, C.-D. Munz, P. L. Roe, and B. J. Sjögreen. On Godunov-type methods near low densities. J. Comp. Phys., 92:273-295, 1991.

[16] C. Farhat, J.-F. Gerbeau, and A. Rallu. FIVER: a finite volume method based on exact twophase Riemann problems and sparse grids for multi-material flows with large density jumps. J. Comp. Phys., 231:6360-6379, 2012.

[17] G. Gallice. Positive and Entropy Stable Godunov-type Schemes for Gas Dynamics and MHD Equations in Lagrangian or Eulerian Coordinates . Numer. Math., 94:673-713, 2003.

[18] P. Germain. Mécanique, volume I. Ellipses, 1986.

[19] M.E. Gurtin, E. Fried, and L. Anand. The Mechanics and Thermodynamics of Continua. Cambridge University Press, 2009.

[20] A. Harten, B. Engquist, S. Osher, and S. Chakravarthy. Uniformly high order essentially non-oscillatory schemes, III. J. Comp. Phys., 71:231-303, 1987.

[21] R. A. Horn and C. R. Johnson. Matrix analysis. Cambridge University Press, 1985.

[22] G.-S. Jiang and C.-W. Shu. Efficient implementation of weighted eno schemes. J. Comp. Phys., 126:202-228, 1996.

[23] J.R. Kamm and F.X. Timmes. On efficient generation of numerically robust Sedov solutions. Technical Report LA-UR-07-2849, Los Alamos National Laboratory, 2007.

[24] G. Kluth and B. Després. Discretization of hyperelasticity on unstructured mesh with a cellcentered Lagrangian scheme. J. Comp. Phys., 229(24):9092-9118, 2010.

[25] B. Van Leer. Towards the ultimate conservative difference scheme. V-A second-order sequel to Godunov's method. J. Comput. Phys., 32:101-136, 1979.

[26] R. J. LeVeque. Finite Volume Methods for Hyperbolic Problems, volume 31. Cambridge Texts in Applied Mathematics, 2002.

[27] W. Liu, J. Cheng, and C.-W. Shu. High order conservative Lagrangian scheme with LaxWendroff type time discretization for the compressible Euler equations. J. Comp. Phys., 228(23):8872-8891, 2009.

[28] X.-D. Liu, S. Osher, and T. Chan. Weighted essentially non-oscillatory schemes. J. Comp. Phys., 115:200-212, 1994.

[29] R. Loubère. Une Méthode Particulaire Lagrangienne de type Galerkin Discontinu. Application à la Mécanique des Fluides et l'Interaction Laser/Plasma. PhD thesis, Université Bordeaux I, 2002. 
[30] P.-H. Maire. Contribution to the numerical modeling of Inertial Confinement Fusion, 2011. Habilitation à Diriger des Recherches, Bordeaux University, available at http://tel . archives-ouvertes.fr/docs/00/58/97/58/PDF/hdr_main.pdf.

[31] P.-H. Maire, R. Abgrall, J. Breil, R. Loubère, and B. Rebourcet. A nominally second-order cell-centered Lagrangian scheme for simulating elastic-plastic flows on two-dimensional unstructured grids. J. Comp. Phys., 235:626-665, 2013.

[32] P.-H. Maire, R. Abgrall, J. Breil, and J. Ovadia. A cell-centered Lagrangian scheme for twodimensional compressible flow problems. SIAM J. Sci. Comput., 29:1781-1824, 2007.

[33] P.-H. Maire and J. Breil. A second-order cell-centered Lagrangian scheme for two-dimensional compressible flow problems. Int. J. Numer. Meth. Fluids, 56:1417-1423, 2008.

[34] P.-H. Maire and B. Nkonga. Multi-scale Godunov-type method for cell-centered discrete Lagrangian hydrodynamics. J. Comp. Phys., 228:799-821, 2009.

[35] C. D. Munz. On Godunov-type schemes for Lagrangian gas dynamics. SIAM J. Numer. Anal., 31:17-42, 1994.

[36] W. F. Noh. Errors for calculations of strong shocks using artificial viscosity and an artificial heat flux. J. Comp. Phys., 72:78-120, 1987.

[37] B. Perthame. Second-order Boltzmann schemes for compressible Euler equations in one and two space dimensions. SIAM J. Numer. Anal., 29:1-19, 1992.

[38] B. Perthame and C.-W. Shu. On positivity preserving finite volume schemes for Euler equations. Numer. Mat., 73:119-130, 1996.

[39] B. J. Plohr and D. H. Sharp. A Conservative Eulerian Formulation of the Equations for Elastic Flows. Advances in Applied Mathematics, 9:481-499, 1988.

[40] C.-W. Shu and S. Osher. Efficient implementation of essentially non-oscillatory shock-capturing schemes. J. Comp. Phys., 77:439-471, 1988.

[41] F. Vilar. Cell-centered discontinuous Galerkin discretization for two-dimensional Lagrangian hydrodynamics. Computers and Fluids, 64:64-73, 2012.

[42] F. Vilar, P.-H. Maire, and R. Abgrall. Cell-centered discontinuous Galerkin discretizations for two-dimensional scalar conservation laws on unstructured grids and for one-dimensional Lagrangian hydrodynamics. Computers and Fluids, 46(1):498-604, 2011.

[43] F. Vilar, P.-H. Maire, and R. Abgrall. A discontinuous Galerkin discretization for solving the two-dimensional gas dynamics equations written under total Lagrangian formulation on general unstructured grids. J. Comp. Phys., 276:188-234, 2014.

[44] F. Vilar, C.-W. Shu, and P.-H. Maire. Positivity-preserving cell-centered Lagrangian schemes for multi-material compressible flows: From first-order to high-orders. Part I: The onedimensional case. J. Comp. Phys., 2015. Submitted.

[45] X. Zhang and C.-W. Shu. On maximum-principle-satisfying high order schemes for scalar conservation laws. J. Comp. Phys., 229:3091-3120, 2010. 
[46] X. Zhang and C.-W. Shu. On positivity preserving high order discontinuous Galerkin schemes for compressible Euler equations on rectangular meshes. J. Comp. Phys., 229:8918-8934, 2010.

[47] X. Zhang and C.-W. Shu. Positivity preserving high order discontinuous Galerkin schemes for compressible Euler equations with source term. J. Comp. Phys., 230:1238-1248, 2011.

[48] X. Zhang and C.-W. Shu. Positivity preserving high order finite difference WENO schemes for compressible Euler equations. J. Comp. Phys., 231:2245-2258, 2012.

[49] X. Zhang, Y. Xia, and C.-W. Shu. Maximum-principle-satisfying and positivity-preserving high order discontinuous galerkin schemes for conservation laws on triangular meshes. J. Sci. Comput., 50:29-62, 2012. 